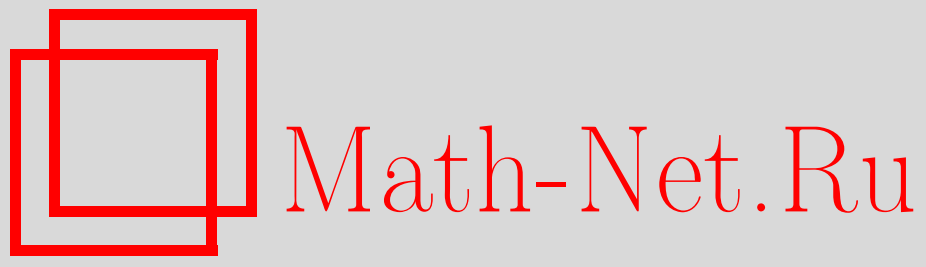

Г. И. Ольшанский, Вероятностные меры на дуальных объектах к компактным симметрическим пространствам и гипергеометрические тождества, Функи. анализ и его прил., 2003, том 37, выпуск 4, 49-73

DOI: https://doi.org/10.4213/faa168

Использование Общероссийского математического портала MathNet.Ru подразумевает, что вы прочитали и согласны с пользовательским соглашением

http://www.mathnet.ru/rus/agreement

Параметры загрузки:

IP : 54.198 .55 .26

26 апреля 2023 г., 17:52:10

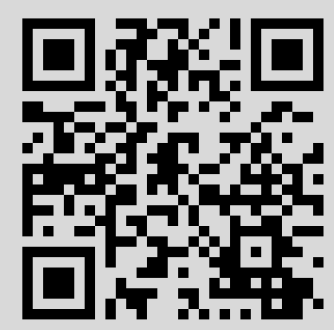


Функциональный анализ и его приложения

2003, т. 37, вып. 4, с. 49-73

УДК 517.986.68+519.117

\title{
Вероятностные меры на дуальных объектах к компактным симметрическим пространствам и гипергеометрические тождества*
}

\author{
(c) 2003. Г. И. ОльШАНСКИЙ
}

Посвящается И. М. Гельфанду в связи с его 90-летием

\section{Введение}

Одной из красивейших формул в классической теории специальных функций является формула суммирования двустороннего гипергеометрического ряда ${ }_{2} \mathrm{H}_{2}$, найденная Дуголлом (1907 г.). Она может быть записана в виде

$$
\begin{aligned}
& \sum_{n=-\infty}^{\infty} \frac{1}{\Gamma(z-n+1) \Gamma\left(z^{\prime}-n+1\right) \Gamma(w+n+1) \Gamma\left(w^{\prime}+n+1\right)} \\
& \quad=\frac{\Gamma\left(z+w+z^{\prime}+w^{\prime}+1\right)}{\Gamma(z+w+1) \Gamma\left(z+w^{\prime}+1\right) \Gamma\left(z^{\prime}+w+1\right) \Gamma\left(z^{\prime}+w^{\prime}+1\right)},
\end{aligned}
$$

см. $[6,1,8]$. Здесь $z, z^{\prime}, w, w^{\prime}-$ комплексные параметры. В настоящей статье обсуждаются многомерные аналоги тождества (0.1), возникающие в некоммутативном гармоническом анализе на бесконечномерных римановых симметрических пространствах. Наши тождества зависят от тех же параметров $z, z^{\prime}, w, w^{\prime}$ и еще от дополнительного параметра $\theta$.

Пусть $\mathscr{G} / \mathscr{K}$ - риманово симметрическое пространство компактного типа. Под дуальнымм объектом к $\mathscr{G} / \mathscr{K}$ мы понимаем множество классов эквивалентности неприводимых представлений группы $\mathscr{G}$, обладающих $\mathscr{K}$-инвариантным вектором. Это счетное множество, которое можно отождествить с множеством $\left\{\Phi_{\nu}\right\}$ неразложимых сферических функций на $\mathscr{G} / \mathscr{K}$; здесь метка $\nu$ пробегает некоторое множество весов $\{\nu\}$. Если задана вероятностная мера $\mathscr{M}$ на множестве $\{\nu\}$, то функция

$$
\psi=\psi_{\mathscr{M}}=\sum_{\nu} \mathscr{M}(\nu) \Phi_{\nu}
$$

есть (разложимая) сферическая функция, т.е. это положительно определенная функция на группе $\mathscr{G}$, двусторонне инвариантная относительно $\mathscr{K}$ и принимающая значение 1 в единице. Обратно, всякая функция $\psi$ с такими свойствами происходит из некоторой вероятностной меры $\mathscr{M}$ на $\{\nu\}$, и мы можем интерпретировать (0.2) как «разложение в ряд Фурье» функции $\psi$.

Предположим, что $\mathscr{G} / \mathscr{K}$ содержит меньшее компактное симметрическое пространство $\mathscr{G}^{\prime} / \mathscr{K}^{\prime}$, причем $\mathscr{G}^{\prime} \subset \mathscr{G}, \mathscr{K}^{\prime} \subset \mathscr{K}$, и пусть $\{\mu\}$ обозначает множество

*Работа частично поддержана грантом CRDF. 
меток, индексирующих неразложимые сферические функции для меньшего пространства. Скажем, что мера $\mathscr{M}$ на $\{\nu\}$ согласована с мерой $\mathscr{M}^{\prime}$ на $\{\mu\}$, если $\psi_{\mathscr{M}}{ }^{\prime}$ совпадает с ограничением функции $\psi_{\mathscr{M}}$ на $\mathscr{G}^{\prime}$. Это условие можно представить в виде

$$
\mathscr{M}^{\prime}(\mu)=\sum_{\nu} q(\mu, \nu) \mathscr{M}(\nu),
$$

где коэффициенты $q(\mu, \nu)$ зависят только от симметрических пространств и определяются из разложения

$$
\Phi_{\nu}{\mid \mathscr{G}^{\prime}}=\sum_{\mu} q(\mu, \nu) \Phi_{\mu} .
$$

Отметим, что коэффициенты $q(\mu, \nu)$ неотрицательны и удовлетворяют соотношению

$$
\sum_{\mu} q(\mu, \nu)=1
$$

при любом фиксированном $\nu$.

Мы будем рассматривать три серии $\{G(N) / K(N)\}$ классических симметрических пространств с корневой системой $A_{N}$, где $N=1,2, \ldots$ :

$$
U(N) / O(N), \quad(U(N) \times U(N)) / U(N), \quad U(2 N) / S p(N) .
$$

Множество меток $\{\nu\}$ для $G(N) / K(N)$ одно и то же для всех трех серий; его можно отождествить с множеством целочисленных $N$-мерных векторов с невозрастающими координатами:

$$
\nu=\left(\nu_{1}, \ldots, \nu_{N}\right) \in \mathbb{Z}^{N}, \quad \nu_{1} \geqslant \cdots \geqslant \nu_{N} .
$$

Это множество будет обозначаться через $\operatorname{SGN}(N)$, а сами векторы $\nu \in \operatorname{SGN}(N)$ будут называться сигнатурами длины $N$. Соответствующие неразложимые сферические функции $\Phi_{\nu}$ можно отождествить с подходящим образом нормализованными многочленами Джека от $N$ переменных, где «джековский параметр» $\theta$ принимает три специальных значения $\frac{1}{2}, 1,2$ соответственно для первой, второй и третьей серии в $(0.6)$.

Если положить $\mathscr{G} / \mathscr{K}=G(N) / K(N)$ и $\mathscr{G}^{\prime} / \mathscr{K}^{\prime}=G(N-1) / K(N-1)$, то соответствующие коэффициенты $q(\mu, \nu)$ можно явно вычислить из (0.4). Более того, если мы возьмем в качестве $\Phi_{\nu}$ (нормализованные) многочлены Джека с произвольным положительным параметром $\theta$, то эти коэффициенты, обозначаемые далее через $q(\mu, \nu ; \theta)$, будут по-прежнему неотрицательными и удовлетворяющими соотношению $(0.5)$. Далее, $q(\mu, \nu ; \theta)$ отличен от нуля тогда и только тогда, когда $\mu \prec \nu$, т. е. выполняются условия перемежаемости Гельфанда-Цетлина

$$
\nu_{1} \geqslant \mu_{1} \geqslant \nu_{2} \geqslant \cdots \geqslant \nu_{N-1} \geqslant \mu_{N-1} \geqslant \nu_{N} .
$$

В настоящей статье изучается некоторое семейство $\left\{M_{N}\left(\cdot \mid z, z^{\prime}, w, w^{\prime} ; \theta\right)\right\}$ вероятностных мер (см. формулы (2.1)-(2.4)). Здесь $N$-я мера определена на пространстве $\mathrm{SGN}(N)$; она зависит от «джековского параметра» $\theta>0$ и комплексных параметров $z, z^{\prime}, w, w^{\prime}$ (на которые налагаются дополнительные условия, обеспечивающие неотрицательность величины $M_{N}\left(\nu \mid z, z^{\prime}, w, w^{\prime} ; \theta\right)$ ).

Мы называем эти меры Zw-мерами. Они родственны так называемым z-мерам на разбиениях, которые возникают в контексте теории представлений бесконечной симметрической группы, см. $[23,14,2,5]$. 
Следующее утверждение (теорема 2.3) является основным результатом рабо-

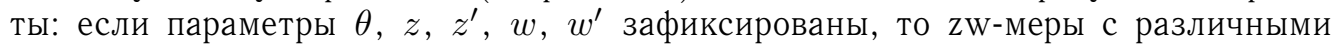
индексами $N$ попарно согласованы, как в $(0.3)$, т. е.

$$
M_{N-1}\left(\mu \mid z, z^{\prime}, w, w^{\prime} ; \theta\right)=\sum_{\nu: \mu \prec \lambda} q(\mu, \nu ; \theta) M_{N}\left(\nu \mid z, z^{\prime}, w, w^{\prime} ; \theta\right) .
$$

Мы называем (0.9) соотношением когерентности для zw-мер. В статье дается (с разной степенью подробности) три доказательства соотношения когерентности. Мы увидим, что это соотношение, равно как и соотношение

$$
\sum_{\nu \in \operatorname{SGN}(N)} M_{N}\left(\nu \mid z, z^{\prime}, w, w^{\prime} ; \theta\right)=1
$$

суть комбинаторные тождества гипергеометрического типа. В простейшем случае $N=1$ оба тождества, $(0.9)$ и $(0.10)$, не зависят от $\theta$ и превращаются в формулу Дуголла (0.1).

Если $\theta$ принимает одно из трех специальных значений $\frac{1}{2}, 1,2$, то ZW-меры $M_{N}$ имеют важный теоретико-представленческий смысл. А именно, последовательность $\left\{M_{N}\right\}$ определяет некоторую разложимую сферическую функцию $\psi$ на бесконечномерном симметрическом пространстве $G / K$, индуктивном пределе конечномерных пространств $G(N) / K(N)$ из соответствующей серии (0.6). Функция $\psi$ зависит от параметров $z, z^{\prime}, w, w^{\prime}$ и определяет приводимое унитарное представление $T$ группы $G$ с выделенным $K$-инвариантным вектором.

Случай $\theta=1$ был подробно изучен в работах Бородина и автора, см. [22] и [4]. В [22] сначала строятся ${ }^{1)}$ представления $T$, а затем из их конструкции находятся $\mathrm{zW}$-меры. В работе [4] описано разложение представлений $T$ на неприводимые, что достигается изучением асимптотического поведения $z W-м е p ~ M_{N}$ в пределе при $N \rightarrow \infty$.

Отметим, что конструкцию работы [22] можно перенести на случаи $\theta=\frac{1}{2}, 2^{2)}$, что приводит к формулам для $\mathrm{zW}$-мер с $\theta=\frac{1}{2}$ и $\theta=2$. Сразу видно, что эти формулы могут быть естественным образом экстраполированы на любые положительные значения параметра $\theta$. Однако для общего $\theta$, когда подход из $[22]$ уже неприменим, вовсе не очевидно, почему соотношения $(0.9),(0.10)$ остаются в силе.

Настоящая работа возникла из попытки лучше понять природу $z w-м e p^{3)}$. Mне хотелось найти прямую и прозрачную конструкцию $\mathrm{zW}$-мер, которая могла бы объяснить соотношения $(0.9)$, (0.10) и была бы пригодна для всех $\theta>0$. Я надеюсь, что конструкция, приведенная в $\$ 3$, удовлетворяет этим требованиям.

Чрезвычайно привлекательная задача состоит в вычислении асимптотики zW-мер с общим параметром $\theta$ при больших $N$. В частном случае $\theta=1$ задача была решена в [4]. Вероятно, усовершенствование метода [4] позволит решить ее и для $\theta=\frac{1}{2}, 2$. Но для других значений параметра $\theta$ задача выглядит го-

1) Идея конструкции восходит к работе Пикрела [24].

2) В связи с этим см. работу Неретина [17].

3) Родственный вопрос касается представлений $T$, изучавшихся в $[22,4]$. Верно ли, что они в каком-то смысле выделены? Что именно они являются «правильными» объектами в задаче гармонического анализа? 
раздо более трудной и требует привлечения новых идей ${ }^{1)}$. Ее можно сравнить с аналогичной задачей для $N$-частичного кругового ансамбля Дайсона [7] с произвольным параметром $\beta=2 \theta$. Возможно, zw-меры следует рассматривать как своего рода двойственные объекты по отношению к круговым ансамблям ${ }^{2}$.

Статья организована следующим образом. В $\$ 1$ напоминаются некоторые факты о многочленах Джека и определяются когерентные системы мер. В §2 вводятся ZW-меры. Поскольку выражение для них выглядит на первый взгляд довольно громоздким, объясняется, как его можно вывести. Это приводит к доказательству соотношения $(0.10)$, но не $(0.9)$. §3 является центральной частью работы. Здесь $\mathrm{zW}$-меры выводятся с помощью некоторой процедуры, которую я называю прямоугольным трюком (она использует прямоугольные диаграммы Юнга и аналитическое продолжение посредством теоремы Карлсона). Как следствие получается доказательство тождеств (0.9) и (0.10). Идея прямоугольного трюка, как мне кажется, весьма прозрачна (она объяснена в начале §3), однако проверка выполнения предпосылок теоремы Карлсона требует технической работы. В $§ 4$ изложено альтернативное доказательство тождеств $(0.9),(0.10)$, состоящее в редукции к некоторой многомерной гипергеометрической формуле суммирования, принадлежащей Густафсону [10]. Второе доказательство занимает мало места, но его простота обманчива: трудность скрыта в весьма нетривиальной формуле Густафсона. Механизм этого доказательства мне пока не вполне ясен. В §5 кратко описана связь Zw-мер с интегралами типа Сельберга ${ }^{3)}$. C ее помощью можно получить еще одно доказательство тождеств $(0.9),(0.10)$. Я привожу несколько доказательств по следующим причинам. Во-первых, мне кажется, что само существование различных комбинаторных подходов к ZW-мерам свидетельствует о содержательности этого понятия. Во-вторых, еще не ясно, какой из имеющихся методов будет работать в других сходных ситуациях.

Настоящая статья может рассматриваться как продолжение статьи Бородина и автора [2]: там неявно содержится идея «прямоугольного трюка». С другой стороны, я хочу с благодарностью отметить влияние, оказанное на меня заметками Керова [12]. В этих заметках, написанных еще в 1993 г., получены первые комбинаторные результаты о ZW-мерах (Керов рассматривал так называемую вырожденную серию $\mathrm{zW}$-мер с параметром $\theta$, равным 1 ).

Отметим, что существуют непрерывные версии zw-мер, для которых выполнены интегральные тождества, аналогичные $(0.10)$ (см. работы Неретина $[17,18])$. $\mathrm{C}$ точки зрения теории представлений этот сюжет связан с переходом к плоской версии симметрических пространств (0.6) и заменой многочленов Джека на так называемые многомерные функции Бесселя, см. [20, §4]. В частном случае $\theta=1$ непрерывный аналог zW-мер подробно рассмотрен в [3].

Я чрезвычайно признателен Юрию Неретину и Эрику Райнсу за ряд важных замечаний. В частности, они указали мне на возможную связь с работами Густафсона.

1) В контексте ZW-мер на разбиениях некоторые результаты для целых значений параметра $\theta$ получены в [5].

2) Сходство zw-мер с вероятностными ансамблями, возникающими в теории случайных матриц, подчеркивалось в [4].

3) Впервые она была открыта Керовым [12]. 


\section{§1. Многочлены Джека и когерентные системы мер на сигнатурах}

В качестве справочного руководства по многочленам Джека мы используем гл. VI книги Макдональда [16]. На протяжении всей статьи $\theta>0$ есть фиксированный параметр. В обозначениях $[16, \S \mathrm{VI} .10] \theta$ есть $k=\alpha^{-1}$. Многочлены Джека с $N$ переменными мы обозначаем через $P_{\nu}\left(u_{1}, \ldots, u_{N} ; \theta\right)$ (что соответствует $P_{\nu}^{(1 / \theta)}\left(u_{1}, \ldots, u_{N}\right)$ в $[16, \S$ VI.10]). Метка $\nu$ пробегает у нас множество $\operatorname{SGN}(N)$ (см. введение). Эти многочлены являются симметрическими многочленами Лорана. Отметим, что Макдональд рассматривает только неотрицательные сигнатуры $\nu$ и, таким образом, только обычные многочлены, но все нужные нам результаты легко распространяются на случай лорановских многочленов благодаря формуле

$$
\begin{gathered}
P_{\nu+m}\left(u_{1}, \ldots, u_{N} ; \theta\right)=\left(u_{1} \ldots u_{N}\right)^{m} P_{\nu}\left(u_{1}, \ldots, u_{N} ; \theta\right), \\
m \in \mathbb{Z}, \nu+m:=\left(\nu_{1}+m, \ldots, \nu_{N}+m\right) .
\end{gathered}
$$

Пусть $\mathbb{T} \subset \mathbb{C}-$ единичная окружность, а $\mathbb{T}^{N}=\mathbb{T} \times \cdots \times \mathbb{T}$ есть $N$-мерный тор. Многочлены Джека ортогональны на $\mathbb{T}^{N}$ относительно меры $(N !)^{-1} W_{N}(u, \theta) d u$, где $u=\left(u_{1}, \ldots, u_{N}\right)$ пробегает $\mathbb{T}^{N}, d u$ есть нормированная инвариантная мера на $\mathbb{T}^{N}$ и

$$
W_{N}(u ; \theta)=\prod_{1 \leqslant i<j \leqslant N}\left|u_{i}-u_{j}\right|^{2 \theta} .
$$

При $\theta=1$ многочлены Джека сводятся к (лорановским) многочленам Шура $s_{\nu}\left(u_{1}, \ldots, u_{N}\right)$, совпадающим с неприводимыми характерами группы $U(N)$.

Мы используем обозначение

$$
\Phi_{\nu}\left(u_{1}, \ldots, u_{N} ; \theta\right)=\frac{P_{\nu}\left(u_{1}, \ldots, u_{N} ; \theta\right)}{P_{\nu}(\underbrace{1, \ldots, 1}_{N} ; \theta)}
$$

для многочленов Джека, нормализованных в точке $(1, \ldots, 1)$.

Имеет место следующее правило ветвления (см. [16, §VI.10]):

$$
P_{\nu}\left(u_{1}, \ldots, u_{N-1}, 1 ; \theta\right)=\sum_{\mu \prec \nu} \psi_{\nu / \mu}(\theta) P_{\mu}\left(u_{1}, \ldots, u_{N-1} ; \theta\right),
$$

где коэффициенты $\psi_{\nu / \mu}(\theta)$ (в обозначениях $[16, \S \mathrm{VI} .10] \psi_{\nu / \mu}^{(1 / \theta)}$ ) строго положительны и зависят рационально от параметра $\theta$. Когда $\theta=1$, коэффициенты равны 1 для всех $\mu \prec \nu$.

В терминах нормализованных многочленов правило ветвления принимает вид

$$
\Phi_{\nu}\left(u_{1}, \ldots, u_{N-1}, 1 ; \theta\right)=\sum_{\mu \prec \nu} q(\mu, \nu ; \theta) \Phi_{\mu}\left(u_{1}, \ldots, u_{N-1} ; \theta\right),
$$

где

$$
q(\mu, \nu ; \theta)=\psi_{\nu / \mu}(\theta) \cdot \frac{P_{\mu}(\overbrace{1, \ldots, 1}^{N-1} ; \theta)}{P_{\nu}(\underbrace{1, \ldots, 1}_{N} ; \theta)} .
$$


Явные выражения для $\psi_{\nu / \mu}(\theta)$ и $P_{\nu}(1, \ldots, 1 ; \theta)$ приведены ниже в $(4.9),(3.55)$ (см. также (2.4)). Поскольку обе эти величины строго положительны, таковы же и числа $q(\mu, \nu ; \theta)$. Ясно также, что для любого фиксированного $\nu$

$$
\sum_{\mu: \mu \prec \nu} q(\mu, \nu ; \theta)=1 .
$$

Следуя общей философии Вершика-Керова [26], мы называем числа $q(\mu, \nu ; \theta)$ копереходными вероятностями. Их явное выражение приведено ниже в (4.8).

Условимся, что множество SGN(0) состоит из единственного элемента, обозначаемого через $\varnothing$, и что $\varnothing \prec \nu$ и $q(\varnothing, \nu ; \theta)=1$ для любого $\nu \in \operatorname{SGN}(1)=\mathbb{Z}$.

ОПРЕДЕЛЕНИЕ 1.1. Предположим, что для каждого $N=0,1,2, \ldots$ задана вероятностная мера $M_{N}$ на множестве $\operatorname{SGN}(N)$. Скажем тогда, что последовательность $M_{0}, M_{1}, \ldots$ образует когерентную систему, если она согласована с копереходными вероятностями, т.е.

$$
M_{N-1}(\mu)=\sum_{\nu \in \operatorname{SGN}(N): \mu \prec \nu} q(\mu, \nu ; \theta) M_{N}(\nu)
$$

для любого $N=1,2, \ldots$ и любого $\mu \in \operatorname{SGN}(N-1)$. Назовем (1.8) соотношением когерентности.

Отметим, что если $M_{N}$ - вероятностная мера на $\operatorname{SGN}(N)$ и $M_{N-1}$ определяется из соотношения когерентности, то $M_{N-1}$ будет вероятностной мерой на $\operatorname{SGN}(N-1)$. Таким образом, в когерентной системе $\left\{M_{N}\right\}$ каждый член $M_{N}$ может рассматриваться как уточнение предыдущего члена $M_{N-1}$.

ЗАмЕчАНИЕ 1.2. Если $\left\{M_{N}\right\}-$ последовательность конечных (возможно, комплексных) мер, удовлетворяющая соотношению когерентности (1.8), причем $M_{0}(\varnothing)=1$, то для любого $N=1,2, \ldots$ выполнено равенство $\sum_{\nu \in \operatorname{SGN}(N)} M_{N}(\nu)$ $=1$. В частности, если каждая $M_{N}$ есть обычная мера, то она является вероятностной мерой.

В самом деле, для любого $N=1,2, \ldots$

$$
\begin{aligned}
\sum_{\mu \in \operatorname{SGN}(N-1)} M_{N-1}(\mu) & =\sum_{\mu \in \operatorname{SGN}(N-1)} \sum_{\nu \in \operatorname{SGN}(N): \mu \prec \nu} q(\mu, \nu ; \theta) M_{N}(\nu) \\
& =\sum_{\nu \in \operatorname{SGN}(N)}\left(\sum_{\mu \in \operatorname{SGN}(N-1): \mu \prec \nu} q(\mu, \nu ; \theta)\right) M_{N}(\nu) \\
& =\sum_{\nu \in \operatorname{SGN}(N)} M_{N}(\nu),
\end{aligned}
$$

где первое равенство следует из (1.8), а третье - из (1.7). Таким образом, сумма $\sum_{\nu \in \operatorname{SGN}(N)} M_{N}(\nu)$ не зависит от $N$. Но для $N=0$ она сводится к $M_{0}(\varnothing)$, что равно 1 по предположению.

\section{§2. zw-меры}

Происхождение приведенных ниже формул объяснено в доказательстве теоремы 2.2. Пусть $z, z^{\prime}, w, w^{\prime}$ суть комплексные параметры. Для $\nu \in \operatorname{SGN}(N)$ 
положим

$$
\begin{aligned}
M_{N}\left(\nu \mid z, z^{\prime}, w, w^{\prime} ; \theta\right) & =\frac{1}{C_{N}\left(z, z^{\prime}, w, w^{\prime} ; \theta\right)} \operatorname{Dim}_{N}(\nu ; \theta) \operatorname{Dim}_{N}^{\prime}(\nu ; \theta) \\
\times & \prod_{i=1}^{N} \frac{1}{\Gamma\left(z-\nu_{i}+(i-1) \theta+1\right) \Gamma\left(z^{\prime}-\nu_{i}+(i-1) \theta+1\right)} \\
\times & \prod_{i=1}^{N} \frac{1}{\Gamma\left(w+\nu_{i}+(N-i) \theta+1\right) \Gamma\left(w+\nu_{i}+(N-i) \theta+1\right)},
\end{aligned}
$$

где $C_{N}\left(z, z^{\prime}, w, w^{\prime} ; \theta\right)$ есть нормировочная константа,

$$
\begin{aligned}
C_{N}\left(z, z^{\prime}, w, w^{\prime} ; \theta\right) & =\prod_{i=1}^{N} \frac{\Gamma\left(z+z^{\prime}+w+w^{\prime}+(i-1) \theta+1\right)}{\Gamma((i-1) \theta+1)} \\
\times & \prod_{i=1}^{N} \frac{1}{\Gamma(z+w+(i-1) \theta+1) \Gamma\left(z+w^{\prime}+(i-1) \theta+1\right)} \\
\times & \prod_{i=1}^{N} \frac{1}{\Gamma\left(z^{\prime}+w+(i-1) \theta+1\right) \Gamma\left(z^{\prime}+w^{\prime}+(i-1) \theta+1\right)},
\end{aligned}
$$

И

$$
\begin{aligned}
& \operatorname{Dim}_{N}(\nu ; \theta)=\prod_{1 \leqslant i<j \leqslant N} \frac{\Gamma\left(\nu_{i}-\nu_{j}+(j-i) \theta+1\right)}{\Gamma\left(\nu_{i}-\nu_{j}+(j-i) \theta+1-\theta\right)} \frac{\Gamma((j-i) \theta+1-\theta)}{\Gamma((j-i) \theta+1)}, \\
& \operatorname{Dim}_{N}^{\prime}(\nu ; \theta)=\prod_{1 \leqslant i<j \leqslant N} \frac{\Gamma\left(\nu_{i}-\nu_{j}+(j-i) \theta+\theta\right)}{\Gamma\left(\nu_{i}-\nu_{j}+(j-i) \theta\right)} \frac{\Gamma((j-i) \theta)}{\Gamma((j-i) \theta+\theta)} .
\end{aligned}
$$

Условимся, что $M_{0}\left(\varnothing \mid z, z^{\prime}, w, w^{\prime} ; \theta\right)=1$ - в согласии с общим принципом, что произведение по пустому множеству равно 1.

Положим

$$
\mathscr{D}=\left\{\left(z, z^{\prime}, w, w^{\prime}\right) \in \mathbb{C}^{4} \mid \operatorname{Re}\left(z+z^{\prime}+w+w^{\prime}\right)>-1\right\} .
$$

Это - (вещественное) полупространство в $\mathbb{C}^{4}$. Далее, положим

$$
\mathscr{D}_{0}=\left\{\left(z, z^{\prime}, w, w^{\prime}\right) \in \mathscr{D} \mid C_{N}\left(z, z^{\prime}, w, w^{\prime} ; \theta\right) \neq 0, N=1,2, \ldots\right\} .
$$

Иными словами, $\mathscr{D}_{0}$ получается удалением из $\mathscr{D}$ четверок $\left(z, z^{\prime}, w, w^{\prime}\right)$, лежащих на (комплексных) гиперплоскостях вида

$$
\begin{aligned}
z+w+1 & =-i-j \theta, & z+w^{\prime}+1 & =-i-j \theta, \\
z^{\prime}+w+1 & =-i-j \theta, & z^{\prime}+w^{\prime}+1 & =-i-j \theta,
\end{aligned}
$$

где $i$ и $j$ пробегают $\{0,1,2, \ldots\}$. Отметим, что всякий компакт в $\mathscr{D}$ может иметь непустое пересечение лишь с конечным числом таких гиперплоскостей. Стало быть, $\mathscr{D}_{0}$ есть открытая область в $\mathbb{C}^{4}$.

ПрЕДЛОЖЕНИЕ 2.1. Для любого $\left(z, z^{\prime}, w, w^{\prime}\right) \in \mathscr{D}_{0}$ величинь $M_{N}\left(\nu \mid z, z^{\prime}, w, w^{\prime} ; \theta\right)$ корректно определень и

$$
\sum_{\nu \in \operatorname{SGN}(N)}\left|M_{N}\left(\nu \mid z, z^{\prime}, w, w^{\prime} ; \theta\right)\right|<\infty, \quad N=1,2, \ldots
$$


Более того, ряды сходятся равномерно на компактах в $\mathscr{D}_{0}$.

ДокАЗАТЕЛЬСтво. Это легко проверяется оценкой отношений гамма-функций. См. доказательство предложения 7.5 в статье автора [22]. (В [22] параметр $\theta$ равен 1 , но для общего $\theta>0$ нужны лишь небольшие очевидные изменения.)

Teоpema 2.2. Пусть $\left(z, z^{\prime}, w, w^{\prime}\right) \in \mathscr{D}_{0}$. Тогда для любого $N=0,1,2, \ldots$

$$
\sum_{\nu \in \operatorname{SGN}(N)} M_{N}\left(\nu \mid z, z^{\prime}, w, w^{\prime} ; \theta\right)=1 .
$$

В силу замечания 1.2 этот факт следует из теоремы 2.3 , сформулированной ниже. Однако мы наметим независимое доказательство, поскольку оно объясняет происхождение формул.

НАБРОСОК ДОКАЗАТЕЛЬСТВА. Введем симметрическую функцию на торе $\mathbb{T}^{N}$, зависящую от комплексных параметров $z, w$ :

$$
f_{z w}\left(u_{1}, \ldots, u_{N}\right)=\prod_{i=1}^{N}\left(1+u_{i}\right)^{z}\left(1+u_{i}^{-1}\right)^{w} .
$$

Чтобы устранить многозначность, мы определяем $(\ldots)^{z}$ или $(\ldots)^{w}$ как голоморфную функцию в $\mathbb{C} \backslash(-\infty, 0]$. Тем самым, строго говоря, наша функция $f_{z w}$ корректно определена только на подмножестве $(\mathbb{T} \backslash\{-1\})^{N} \subset \mathbb{T}^{N}$. Условимся, что $f_{z w}$ равна 0 на дополнении к этому подмножеству. Отметим, что функция $f_{z w}$ интегрируема на всем торе, если $\operatorname{Re}(z+w)>-1$, и непрерывна, если $\operatorname{Re}(z+w)>0$.

Справедлива формула (см. конец доказательства)

$$
f_{z w}\left(u_{1}, \ldots, u_{N}\right)=\sum_{\nu \in \operatorname{SGN}(N)} c(\nu ; z, w ; \theta) P_{\nu}\left(u_{1}, \ldots, u_{N} ; \theta\right),
$$

где

$$
\begin{aligned}
& c(\nu ; z, w ; \theta)=\prod_{i=1}^{N} \frac{\Gamma(z+w+(i-1) \theta+1)}{\Gamma\left(z-\nu_{i}+(i-1) \theta+1\right) \Gamma\left(w+\nu_{i}+(N-i) \theta+1\right)} \\
& \quad \times \prod_{1 \leqslant i<j \leqslant N} \frac{\Gamma((j-i) \theta+1-\theta)}{\Gamma((j-i) \theta+1)} \cdot \prod_{i=1}^{N} \Gamma((i-1) \theta+1) \\
& \times \prod_{1 \leqslant i<j \leqslant N} \frac{\Gamma\left(\nu_{i}-\nu_{j}+(j-i) \theta+1\right)}{\Gamma\left(\nu_{i}-\nu_{j}+(j-i) \theta+1-\theta\right)} .
\end{aligned}
$$

Если $\operatorname{Re}(z+w)$ достаточно велико, то $f_{z w}$ квадратично интегрируема на тоpe $\mathbb{T}^{N}$ с весовой функцией $W_{N}(u ; \theta)$, определенной в $(1.2)$, и ряд сходится. Пусть $(\cdot, \cdot)_{\theta, N}$ обозначает скалярное произведение в гильбертовом пространстве $L^{2}\left(\mathbb{T}^{N},(N !)^{-1} W_{N}(\cdot ; \theta)\right)$. Предполагая $\operatorname{Re}(z+w)$ достаточно большим, имеем

$$
\left(f_{z w}, f_{z w}\right)_{\theta, N}=\sum_{\nu \in \operatorname{SGN}(N)}|c(\nu ; z, w ; \theta)|^{2}\left(P_{\nu}, P_{\nu}\right)_{\theta, N},
$$

так что

$$
\sum_{\nu \in \operatorname{SGN}(N)} \frac{|c(\nu ; z, w ; \theta)|^{2}\left(P_{\nu}, P_{\nu}\right)_{\theta, N}}{\left(f_{z w}, f_{z w}\right)_{\theta, N}}=1 .
$$


Заметим, что

$$
f_{z w}(u) \overline{f_{z w}(u)}=f_{z+\bar{w}, \bar{z}+w}(u), \quad u \in \mathbb{T}^{N}
$$

Отсюда

$$
\begin{aligned}
\left(f_{z w}, f_{z w}\right)_{\theta, N} & =\left(f_{z+\bar{w}, \bar{z}, w}, \mathbf{1}\right)_{\theta, N} \\
& =\left(f_{z+\bar{w}, \bar{z}+w}, P_{\underline{0}}\right)_{\theta, N}=c(\underline{0} ; z+\bar{w}, \bar{z}+w ; \theta)\left(P_{\underline{0}}, P_{\underline{0}}\right)_{\theta, N},
\end{aligned}
$$

где 1 обозначает функцию на $\mathbb{T}^{N}$, тождественно равную 1 , а $\underline{0} \in \mathrm{SGN}(N)$ есть нулевая сигнатура.

$$
\begin{aligned}
& \text { Далее, согласно [16, Ch. VI, (10.38)], } \\
& \left(P_{\nu}, P_{\nu}\right)_{\theta, N}=\prod_{1 \leqslant i<j \leqslant N} \frac{\Gamma\left(\nu_{i}-\nu_{j}+(j-i) \theta+\theta\right) \Gamma\left(\nu_{i}-\nu_{j}+(j-i) \theta+1-\theta\right)}{\Gamma\left(\nu_{i}-\nu_{j}+(j-i) \theta\right) \Gamma\left(\nu_{i}-\nu_{j}+(j-i) \theta+1\right)} .
\end{aligned}
$$

Используя эти формулы и явное выражение для коэффициентов $c(\nu ; z, w ; \theta)$, данное в (2.12), мы можем убедиться в том, что тождество (2.14) совпадает с

$$
\sum_{\nu \in \operatorname{SGN}(N)} M_{N}(\nu ; z, \bar{z}, w, \bar{w} ; \theta)=1 .
$$

Таким образом, мы получаем частный случай теоремы 2.2: $z^{\prime}=\bar{z}, w^{\prime}=\bar{w}$, и напомним еще, что мы предположили, что $\operatorname{Re}(z+w)$ достаточно велико. Чтобы вывести отсюда утверждение теоремы 2.2 в общем виде, достаточно сделать аналитическое продолжение по параметрам.

Остается доказать (2.12). В частном случае $\theta=1$ коэффициенты разложения (2.11) были вычислены в статье автора [22]. Но способ, которым это было сделано, не годится для многочленов Джека, так что мы должны применить другой подход.

Предположим сначала, что $w=0$. Тогда выражение для $c(\nu ; z, w ; \theta)$ обращается в нуль при нарушении условия $\nu_{N} \geqslant 0$, что обусловлено членом

$$
\left.\Gamma\left(w+\nu_{i}+(N-i) \theta+1\right)\right|_{i=N, w=0}=\Gamma\left(\nu_{N}+1\right)
$$

в знаменателе первого произведения в (2.12). Стало быть, суммирование в действительности берется по сигнатурам $\nu$ с неотрицательными координатами, иначе говоря, по разбиениям длины, меньшей или равной $N$.

С другой стороны,

$$
\left.f_{z w}\left(u_{1}, \ldots, u_{N}\right)\right|_{w=0}=\prod_{i=1}^{N}\left(1+u_{i}\right)^{z} .
$$

Разложение этой функции в ряд по многочленам Джека, индексированным разбиениями, хорошо известно. В самом деле, это частный случай биномиальной формулы, см. [20]. Сравнивая наше выражение для коэффициентов с тем, что дается биномиальной формулой, мы заключаем, что формула (2.12) верна для $w=0$.

Далее, предположим, что $w \in \mathbb{Z}$. Тогда

$$
f_{z w}\left(u_{1}, \ldots, u_{N}\right)=\left(u_{1} \ldots u_{N}\right)^{-w} \cdot \prod_{i=1}^{N}\left(1+u_{i}\right)^{z+w} .
$$


Отсюда следует, что коэффициент при $P_{\nu}$ в $f_{z w}$ равен коэффициенту при $P_{\nu+(w, \ldots, w)}$ в $f_{z+w, 0}$. С другой стороны, из выражения $(2.12)$ для $c(\nu ; z, w ; \theta)$ сразу видно, что

$$
c(\nu ; z, w ; \theta)=c(\nu+(w, \ldots, w) ; z+w, 0 ; \theta), \quad w \in \mathbb{Z} .
$$

Следовательно, формула $(2.12)$ верна и для $w \in \mathbb{Z}$.

Наконец, можно доказать, что

$$
c(\nu ; z, w ; \theta)=\frac{\left(f_{z w}, P_{\nu}\right)_{\theta, N}}{\left(P_{\nu}, P_{\nu}\right)_{\theta, N}},
$$

используя теорему Карлсона, сформулированную ниже в §3. Для применения теоремы Карлсона необходимо проверить выполнение ее предпосылок, что требует некоторой технической работы.

Tеорема 2.3. Пусть $\left(z, z^{\prime}, w, w^{\prime}\right) \in \mathscr{D}_{0}$. Тогда величины $M_{N}\left(\nu \mid z, z^{\prime}, w, w^{\prime} ; \theta\right)$, где $N=0,1,2, \ldots$, удовлетворяют соотношению когерентности (0.9).

Два различных доказательства даны в $\$ 3$ и $§ 4$ и еще одно намечено в 55 .

Следствие 2.4. Если для $\left(z, z^{\prime}, w, w^{\prime}\right) \in \mathscr{D}_{0}$ все числа $M_{N}\left(\nu \mid z, z^{\prime}, w, w^{\prime} ; \theta\right)$ вещественны и неотрицательны, то $\left\{M_{N}\left(\cdot \mid z, z^{\prime}, w, w^{\prime} ; \theta\right)\right\}_{N=0,1,2, \ldots}$ есть когерентная система вероятностных мер.

ПримеР 2.5. Вот некоторые достаточные условия на параметры, обеспечивающие неотрицательность чисел $M_{N}\left(\nu \mid z, z^{\prime}, w, w^{\prime} ; \theta\right)$ (дальнейшие подробности для случая $\theta=1$ можно найти в статье автора $[22, \S 7])$.

- Основная серия: $z, w \in \mathbb{C} \backslash \mathbb{Z}, z^{\prime}=\bar{z}, w^{\prime}=\bar{w}$. Условие $\left(z, z^{\prime}, w, w^{\prime}\right) \in \mathscr{D}_{0}$ означает, что $\operatorname{Re}(z+w)>-\frac{1}{2}$.

- Дополнительная серия. Пусть для простоты $\theta=1$. Тогда условия таковы: все параметры вещественны, и существуют такие целые $m, n$, что $m<z, z^{\prime}<$ $m+1$ и $n<w, w^{\prime}<n+1$. Дополнительно предполагаем, что $m+n \geqslant 0$, и это обеспечивает включение $\left(z, z^{\prime}, w, w^{\prime}\right) \in \mathscr{D}_{0}$. Более общо, аналогичные условия существуют, если $\theta$ рационально.

- Вырожденная серия: требуется, чтобы существовали целые $r, s$ с $r+s \geqslant 0$, такие, что $z=r, z^{\prime}>r-1, w=s, w^{\prime}>s-1$ (ввиду очевидной симметрии возможны также перестановки $\left.z \leftrightarrow z^{\prime}, w \leftrightarrow w^{\prime}\right)$. Отметим, что в предыдущих примерах $M_{N}\left(\nu \mid z, z^{\prime}, w, w^{\prime} ; \theta\right)$ всегда строго положительно, тогда как теперь $M_{N}\left(\nu \mid z, z^{\prime}, w, w^{\prime} ; \theta\right)$ обращается в нуль при нарушении неравенств $\nu_{1} \leqslant r$, $\nu_{N} \geqslant-s$. Таким образом, каждая мера $M_{N}\left(\cdot \mid z, z^{\prime}, w, w^{\prime} ; \theta\right)$ сосредоточена на конечном множестве сигнатур $\nu \in \operatorname{SGN}(N)$.

- Смешанные условия: мы можем выбирать различные условия, перечисленные выше, отдельно для $\left(z, z^{\prime}\right)$ и для $\left(w, w^{\prime}\right)$.

\section{§3. Доказательство теоремы 2.3 , основанное на «прямоугольном трюке»}

ИДЕЯ ДОКАЗАТЕЛЬСТВА. Сначала обобщим определение величин $q(\mu, \nu ; \theta)$, данное в (1.5)-(1.6). Предположим, что $\nu \in \operatorname{SGN}(N), \lambda \in \operatorname{SGN}(L)$, где $L>N$. Определим числа $\hat{q}(\nu, \lambda ; \theta)$ как коэффициенты в разложении

$$
\Phi_{\lambda}(u_{1}, \ldots, u_{N}, \underbrace{1, \ldots, 1}_{L-N} ; \theta)=\sum_{\nu \in \operatorname{SGN}(N)} \hat{q}(\nu, \lambda ; \theta) \Phi_{\nu}\left(u_{1}, \ldots, u_{N}\right) .
$$


Ясно, что $\hat{q}(\nu, \lambda ; \theta)=q(\nu, \lambda ; \theta)$ для $L=N+1$, а для $L>N+1$ справедливо рекуррентное соотношение

$$
\hat{q}(\nu, \lambda ; \theta)=\sum_{\kappa: \nu \prec \kappa} q(\nu, \kappa ; \theta) \hat{q}(\kappa, \lambda ; \theta) .
$$

Отсюда следует. что если мы зафиксируем $\lambda$ и положим

$$
\widetilde{M}_{N}(\nu \mid \lambda)=\hat{q}(\nu, \lambda ; \theta), \quad \nu \in \operatorname{SGN}(N), \lambda \in \operatorname{SGN}(L), 0 \leqslant N<L,
$$

то получим усеченное семейство $\left\{\widetilde{M}_{N}\right\}$ вероятностных мер, удовлетворяющих соотношению когерентности до уровня $L-1$, т. е.

$$
\widetilde{M}_{N-1}(\mu \mid \lambda)=\sum_{\nu: \mu \prec \nu} q(\mu, \nu ; \theta) \widetilde{M}_{N}(\nu \mid \lambda), \quad N<L .
$$

Теперь мы возьмем в качестве $\lambda$ сигнатуру специального вида,

$$
\lambda=(\underbrace{r, \ldots, r}_{k}, \underbrace{-s, \ldots,-s}_{l}) \in \operatorname{SGN}(L), \quad r, s, k, l \in \mathbb{Z}_{+}, L=k+l .
$$

Оказывается, тогда

$$
\widetilde{M}_{N}(\nu \mid \lambda)=\left.M_{N}\left(\nu \mid z, z^{\prime}, w, w^{\prime} ; \theta\right)\right|_{z=r, z^{\prime}=-s-k \theta, w=s, w^{\prime}=-r-l \theta},
$$

так что соотношение когерентности

$$
M_{N-1}\left(\mu \mid z, z^{\prime}, w, w^{\prime} ; \theta\right)=\sum_{\nu: \nu \prec \nu} q(\mu, \nu ; \theta) M_{N}\left(\nu \mid z, z^{\prime}, w, w^{\prime} ; \theta\right)
$$

будет иметь место, коль скоро $N<L=k+l$ и

$$
\left(z, z^{\prime}, w, w^{\prime}\right)=(r,-s-k \theta, s,-r-l \theta), \quad r, s, k, l \in \mathbb{Z}_{+} .
$$

Следует отметить, что хотя эта четверка $\left(z, z^{\prime}, w, w^{\prime}\right)$ не лежит в области $\mathscr{D}_{0}$, правая часть в (3.6) имеет смысл.

Затем мы можем доказать, что соотношение (3.7) остается в силе для комплексных значений параметров $z, z^{\prime}, w, w^{\prime}$, используя аналитическое продолжение. Это делается в два этапа. На первом мы фиксируем $z=r \in \mathbb{Z}_{+}, w=s \in \mathbb{Z}_{+}$ и замечаем, что обе стороны соотношения (3.7) зависят от $\left(z^{\prime}, w^{\prime}\right)$ рационально. Это позволяет распространить соотношение на комплексные значения параметров $z^{\prime}, w^{\prime}$. На втором этапе мы избавляемся от условий целочисленности параметров $z, w$. Это более тонкое место, и здесь мы применяем теорему Карлсона.

Перейдем к реализации изложенного плана. Мы будем рассуждать в обратном порядке.

Редукция к случаю $\boldsymbol{z}, \boldsymbol{w} \in \mathbb{Z}_{+}$. Пусть $\left(z, z^{\prime}, w, w^{\prime}\right) \in \mathscr{D}_{0}$, где область $\mathscr{D}_{0} \subset$ $\mathbb{C}^{4}$ определена в (2.6). Наша цель - дать (условное) доказательство тождеств

$$
\begin{gathered}
\sum_{\nu=n \in \mathbb{Z}} M_{1}\left(\nu \mid z, z^{\prime}, w, w^{\prime} ; \theta\right)=1, \\
\sum_{\nu \in \operatorname{SGN}(N): \mu \prec \nu} q(\mu, \nu ; \theta) M_{N}\left(\nu \mid z, z^{\prime}, w, w^{\prime} ; \theta\right)=M_{N-1}\left(\mu \mid z, z^{\prime}, w, w^{\prime} ; \theta\right)
\end{gathered}
$$

(где $N \geqslant 2$ и $\mu \in \operatorname{SGN}(N-1))$ в предположении, что они справедливы в случае, когда $z, w \in \mathbb{Z}_{+}$. 
Зафиксируем произвольным образом $z^{\prime}, w^{\prime} \in \mathbb{C}$ так, чтобы $-\left(z^{\prime}+w^{\prime}+1\right)$ не лежало в $\mathbb{Z}_{+}+\mathbb{Z}_{+} \theta$, и будем считать $z, w$ переменными. По определению области $\mathscr{D}_{0}$ (см. (2.6)) четверка $\left(z, z^{\prime}, w, w^{\prime}\right)$ попадет в $\mathscr{D}_{0}$, коль скоро $\operatorname{Re} z$ и $\operatorname{Re} w$ будут достаточно велики. Напомним, что ряды в левых частях сходятся абсолютно и равномерно на компактах в области $\mathscr{D}_{0}$ (в самом деле, это следует из предложения 2.1). Поскольку левые и правые части наших соотношений суть голоморфные функции в области $\mathscr{D}_{0}$, достаточно доказать соотношения для больших значений $\operatorname{Re} z, \operatorname{Re} w$. Стандартным средством для продолжения тождеств с целых значений параметра на комплексные является теорема единственности Карлсона для аналитических функций:

ТЕОРЕМА КАРЛСОНА. Пусть $\phi(z)-$ голоморфная функция в полуплоскости $\operatorname{Re} z>m-\varepsilon$, где $m \in \mathbb{Z} u \varepsilon>0$ фиксированы. Далее, предположим, ито в этой полуплоскости ф удовлетворяет оценке вида

$$
|\phi(z)| \leqslant \text { const } \exp (B \operatorname{Re} z+A|\operatorname{Im} z|), \quad B>0, A<\pi .
$$

Тогда если ф обращается в нуль в цельх точках $m, m+1, m+2, \ldots$, mо $\phi \equiv 0$.

Подчеркнем важность предположения $A<\pi$. Если $A \geqslant \pi$, то теорема теряет силу: контрпример доставляется функцией $\phi(z)=\sin (\pi z)$.

ДоказатЕЛьство. См. [1, Corollary 10.12.5]. Более сильная версия дана, например, в [9, теорема 2.8.1].

Чтобы проверить (3.11) в нашей конкретной ситуации, нам понадобится несколько оценок.

Лемма 3.1. Пусть $a, b \in \mathbb{C}$ фиксировань, а $n$ пробегает $\mathbb{Z}$. Тогда $1 /(\Gamma(a-$ $n) \Gamma(b+n))$ имеет, самое большее, полиномиальный рост по $n$ nри $n \rightarrow \pm \infty$.

ДокАЗАТЕЛЬство. Пусть $n \rightarrow+\infty$. Если $a \in \mathbb{Z}$, то наше выражение обращается в нуль для достаточно больших $n$ и доказывать нечего. Предположим, что $a \notin \mathbb{Z}$. Тогда

$$
\begin{aligned}
\frac{1}{\Gamma(a-n) \Gamma(b+n)} & =(-1)^{n} \frac{\sin (\pi a)}{\pi} \frac{\Gamma(1-a+n)}{\Gamma(b+n)} \\
& =(-1)^{n} \frac{\sin (\pi a)}{\pi} n^{1-a-b}\left(1+O\left(\frac{1}{n}\right)\right) .
\end{aligned}
$$

Здесь мы воспользовались известной асимптотической формулой

$$
\begin{gathered}
\frac{\Gamma\left(\text { const }_{1}+z\right)}{\Gamma\left(\text { const }_{2}+z\right)}=z^{\text {const }_{1}-\text { const }_{2}}\left(1+O\left(\frac{1}{z}\right)\right), \quad|z| \rightarrow \infty \\
\text { при }-\pi+\delta<\arg z<\pi-\delta, \text { где } \delta>0,
\end{gathered}
$$

см. $[8,1.19(5)]$.

Когда $n \rightarrow-\infty$, рассуждение такое же.

Лемма 3.2. Пусть $а \in \mathbb{C}$ фиксировано, $n$ пробегает $\mathbb{Z} u b$ пробегает фиксированную правую полуплоскость вида

$$
\operatorname{Re} b \geqslant-\operatorname{Re} a-1+p+\varepsilon, \quad p=0,1,2 \ldots, \varepsilon>0 .
$$

тогда существует равномерная по п оценка вида

$$
\left|\frac{\Gamma(a+b+1)}{\Gamma(a+1-n) \Gamma(b+1+n)}\right| \leqslant \operatorname{const}(1+|n|)^{-p} \exp \left(B \operatorname{Re} b+\left(\frac{\pi}{2}+\delta\right)|\operatorname{Im} b|\right),
$$


где $B>0$ - некоторая константа, а $\delta>0$ можно выбрать сколь угодно малой.

ДокАЗАТЕЛЬСтво. Прежде всего, отметим, что если $n$ фиксировано, то рост по $b$, самое большее, полиномиальный, как это видно из (3.13). Более того, если предположить, что $n$ ограничено снизу (т. е. $n \rightarrow+\infty)$, то справедлива гораздо более сильная оценка, чем (3.15). Таким образом, основная трудность в утверждении леммы связана с режимом $n \rightarrow-\infty$.

Мы воспользуемся интегральным представлением

$$
\begin{aligned}
& \frac{\Gamma(a+b+1)}{\Gamma(a+1-n) \Gamma(b+1+n)} \\
& \quad=\frac{1}{\pi} 2^{a+b} \exp \left(-\frac{i \pi}{2}(b-a+2 n)\right) \int_{0}^{\pi}(\sin x)^{a+b} \exp (i(b-a+2 n) x) d x,
\end{aligned}
$$

см. [8, $1.5(29)]$. (Я благодарен Юрию Неретину за совет применить (3.16).)

Предположим сначала, что $p=0$. Имеем

$$
\left|\frac{1}{\pi} 2^{a+b} \exp \left(-\frac{i \pi}{2}(b-a+2 n)\right)\right| \leqslant \operatorname{const} \exp \left(B \operatorname{Re} b+\frac{\pi}{2} \operatorname{Im} b\right)
$$

с подходящим $B$. Далее, для $0 \leqslant x \leqslant \pi$

$$
\begin{gathered}
\left|(\sin x)^{a+b}\right| \leqslant(\sin x)^{\operatorname{Re}(a+b)} \leqslant(\sin x)^{\varepsilon-1}, \\
|\exp (i(b-a+2 n) x)| \leqslant \text { const } \exp (-x \cdot \operatorname{Im} b) .
\end{gathered}
$$

Поскольку функция $(\sin x)^{\varepsilon-1}$ интегрируема, остается оценить

$$
\exp \left(\left(\frac{\pi}{2}-x\right) \operatorname{Im} b\right), \quad 0 \leqslant x \leqslant \pi .
$$

Так как $-\frac{\pi}{2} \leqslant \frac{\pi}{2}-x \leqslant \frac{\pi}{2}$, выражение (3.20) не превосходит $\exp \left(\frac{\pi}{2}|\operatorname{Im} b|\right)$. Таким образом, в случае $p=0$ мы получаем требуемую оценку $(3.15)$, даже с $\delta=0$.

Чтобы разобрать случай, когда $p=1,2, \ldots$, запишем подынтегральное выражение в (3.16) как $F(x) \exp (2 i n x)$, где

$$
F(x)=(\sin x)^{a+b} \exp (i(b-a) x) .
$$

Предположение $\operatorname{Re}(a+b)>p-1+\varepsilon$ гарантирует, что функция $F(x)$ обращается в нуль в $x=0$ и в $x=\pi$ вместе со своими производными до порядка $p-1$, тогда как ее производная порядка $p$ интегрируема. Не нарушая общности, можно предполагать, что $n \neq 0$. Интегрирование по частям $p$ раз дает

$$
\int_{0}^{\pi}(\sin x)^{a+b} \exp (i(b-a+2 n) x) d x=\frac{(-1)^{p}}{(2 i n)^{p}} \int_{0}^{\pi} F^{(p)}(x) \exp (2 i n x) d x .
$$

Более того, справедлива оценка вида

$$
\left|F^{(p)}(x)\right| \leqslant \operatorname{const}(1+|b|)^{p}(\sin x)^{\varepsilon-1} \exp (-x \cdot \operatorname{Im} b) .
$$

Рассуждая так же, как в случае $p=0$, получаем, что отсюда следует требуемая оценка, где малая $\delta>0$ служит для компенсации растущего множителя $(1+|b|)^{p}$ в $(3.23)$. 
УСЛОВНОЕ ДОКАЗАТЕЛЬСТВО тОЖДЕСТВА (3.9). Идея такова. Зафиксируем сначала достаточно большое $z \in \mathbb{Z}_{+}$и будем рассматривать левую часть в (3.9) минус 1 как функцию от $w$, скажем $\phi(w)$. Мы знаем, что $\phi(w)$ голоморфна в правой полуплоскости и обращается в нуль в целых точках (напомним, что обращение в нуль имеет место в силу предположения, которое в действительности будет проверено позже). Используя теорему Карлсона, мы получаем, что $\phi(w) \equiv 0$. Затем мы фиксируем $w$, делая переменным $z$, и снова применяем теорему Карлсона. Для реализации этого рассуждения мы должны только убедиться в справедливости оценки (3.11) (сначала для переменной $w$, а затем для переменной $z$ ).

Чтобы это сделать, выпишем подробно $M_{1}\left(n \mid z, z^{\prime}, w, w^{\prime}\right)$ :

$$
\begin{aligned}
M_{1}\left(n \mid z, z^{\prime}, w, w^{\prime}\right)= & \frac{\Gamma\left(z^{\prime}+w^{\prime}+1\right)}{\Gamma\left(z^{\prime}-n+1\right) \Gamma\left(w^{\prime}+n+1\right)} \frac{\Gamma(z+w+1)}{\Gamma(z-n+1) \Gamma(w+n+1)} \\
& \times \frac{\Gamma\left(z+w^{\prime}+1\right) \Gamma\left(z^{\prime}+w+1\right)}{\Gamma\left(z+w+z^{\prime}+w^{\prime}+1\right)} .
\end{aligned}
$$

Оценим три дроби в правой части (3.24) по отдельности.

Первая дробь не зависит от $z$ и $w$. По лемме 3.1 она растет полиномиально при $n \rightarrow \pm \infty$.

Ко второй дроби применяем лемму 3.2. Поскольку мы вправе считать $\operatorname{Re} z$ и $\operatorname{Re} w$ достаточно большими, мы можем взять $p$ настолько большим, насколько это потребуется для компенсации роста первой дроби по $n$.

Третья дробь не зависит от $n$. Ее рост, когда одна из переменных $z, w$ фиксирована, а другая пробегает некоторую правую полуплоскость, от силы полиномиален (в самом деле, это вытекает из (3.13)).

Это показывает, что предпосылки теоремы Карлсона выполнены на каждом шаге.

УСЛОВНОЕ ДОКАЗАТЕЛЬСТВО ТОЖДЕСТВА (3.10). ЗаПишем $M_{N}\left(\nu \mid z, z^{\prime}, w, w^{\prime} ; \theta\right)$ в виде

$$
\begin{aligned}
\operatorname{Dim}_{N}(\nu ; \theta) \operatorname{Dim}_{N}^{\prime}(\nu ; \theta) \\
\quad \times \prod_{i=1}^{N} \frac{\Gamma\left(z^{\prime}+w^{\prime}+(i-1) \theta+1\right)}{\Gamma\left(z^{\prime}-\nu_{i}+(i-1) \theta+1\right) \Gamma\left(w^{\prime}+\nu_{i}+(N-i) \theta+1\right.} \\
\quad \times \prod_{j=2}^{N-1} \frac{\Gamma(z+w+(j-1) \theta+1)}{\Gamma\left(z-\nu_{j}+(j-1) \theta+1\right) \Gamma\left(w+\nu_{j}+(N-j) \theta+1\right)} \\
\quad \times \prod_{i=1}^{N} \frac{\Gamma\left(z+w^{\prime}+(i-1) \theta+1\right) \Gamma\left(z^{\prime}+w+(i-1) \theta+1\right) \Gamma((i-1) \theta+1)}{\Gamma\left(z+z^{\prime}+w+w^{\prime}+(i-1) \theta+1\right)} \\
\quad \times \frac{\Gamma(z+w+1)}{\Gamma\left(z-\nu_{1}+1\right) \Gamma\left(w+\nu_{1}+(N-1) \theta+1\right)} \\
\quad \times \frac{\Gamma(z+w+(N-1) \theta+1)}{\Gamma\left(z-\nu_{N}+(N-1) \theta+1\right) \Gamma\left(w+\nu_{N}+1\right)}
\end{aligned}
$$

Напомним, что $N \geqslant 2$, а на $\nu$ наложено условие $\mu \prec \nu$, где сигнатура $\mu \in \operatorname{SGN}(N-1)$ фиксирована. Отсюда следует, что $\nu_{1}$ ограничено снизу, $\nu_{N}$ 
ограничено сверху, а при $N>2$ каждая из координат $\nu_{2} \ldots, \nu_{N-1}$ пробегает конечное подмножество в $\mathbb{Z}$.

Как и в приведенном выше доказательстве для (3.9), рассуждение разбивается на два шага: сначала мы фиксируем $z$ и делаем аналитическое продолжение по $w$, затем фиксируем $w$ и делаем то же самое по отношению к $z$.

Опишем необходимые оценки. Прежде всего, величина $q(\mu, \nu ; \theta)$ в $(3.10)$ лежит между 0 и 1 для любой сигнатуры $\nu$, так что она не вызывает проблем.

Выражения (3.25) и (3.26) не зависят от $z, w$, и мы должны только проконтролировать их рост по $\nu$ (а на самом деле только по двум координатам $\left(\nu_{1}, \nu_{N}\right)$, которые не ограничены). Очевидно, что (3.25) растет полиномиально, а (3.26) также растет полиномиально в силу леммы 3.1 .

Выражение (3.27) не зависит от $\left(\nu_{1}, \nu_{N}\right)$. Его рост по переменной $z$ или $w$ (меняющейся в некоторой правой полуплоскости), самое большее, полиномиален. То же верно для (3.28).

Таким образом, если z зафиксировано, то произведение выражений (3.25)(3.28) имеет, самое большее, полиномиальный рост по $\left(w, \nu_{1}, \nu_{N}\right)$, и то же самое верно, если фиксировано $w$ и меняется $\left(z, \nu_{1}, \nu_{N}\right)$.

Остается разобрать (3.29) и (3.30).

Шаг 1. Предположим, что $z$ - фиксированное целое число, откуда следует, что (3.29) обращается в нуль для больших значений $\nu_{1}$ ввиду множителя $\Gamma\left(z-\nu_{1}+1\right)$ в знаменателе. Следовательно, $\nu_{1}$ на самом деле пробегает некоторое конечное множество, так что нам нет нужды контролировать поведение выражения (3.29) по отношению к $\nu_{1}$. Что же касается роста выражения (3.29) по отношению к переменной $w$ (меняющейся в некоторой правой полуплоскости), то он, самое большее, полиномиален. Стало быть, остается оценить (3.30). Здесь мы применяем лемму 3.2 с $n=\nu_{N}$. Как и в доказательстве для (3.9), мы располагаем возможностью взять $p$ достаточно большим, что позволяет компенсировать полиномиальный рост по $\nu_{N}$ остальных выражений.

Шаг 2. Предположим теперь, что $w$ фиксировано, тогда как $z$ меняется в правой полуплоскости Re $z \geqslant$ const, где константа может быть взята сколь угодно большой. Здесь уже и $\nu_{1}$, и $\nu_{N}$ не ограничены. Перепишем $(3.29)$ в виде

$$
\frac{\Gamma(z+w+1)}{\Gamma(z+w+(N-1) \theta+1)} \cdot \frac{\Gamma(z+w+(N-1) \theta+1)}{\Gamma\left(z-\nu_{1}+1\right) \Gamma\left(w+\nu_{1}+(N-1) \theta+1\right)} .
$$

Первая дробь не зависит от $\nu_{1}$ и имеет от силы полиномиальный рост по $z$. Ко второй дроби мы применяем лемму 3.2 с $a=w+N-1, b=z, n=-\nu_{1}$ и берем $p$ настолько большим, чтобы компенсировать рост по $\nu_{1}$ остальных выражений. Наконец, обратимся к (3.30). Здесь мы не можем снова применить лемму 3.2 , поскольку это дало бы (вместе с предыдущей оценкой для (3.29)) множитель $\exp (\pi|\operatorname{Im} z|)$, который не согласуется с предпосылками теоремы Карлсона. Однако эту трудность можно преодолеть с помощью иной оценки, которая основана на том факте, что $\nu_{N}$ ограничено сверху.

В самом деле, можно предполагать, что $w$ не является целым (в противном случае (3.30) обращалось бы в нуль при больших отрицательных $\left.\nu_{N}\right)$. Зафиксируем большое натуральное число $q$ (оно будет компенсировать рост по $\nu_{N}$ 
остальных множителей) и положим $n=-\nu_{N}$ в (3.30). Без ограничения общности можно предполагать, что $n$ пробегает $\{1,2, \ldots\}$. Тогда

$$
\begin{gathered}
\frac{\Gamma(z+w+(N-1) \theta+1)}{\Gamma\left(z-\nu_{N}+(N-1) \theta+1\right) \Gamma\left(w+1+\nu_{N}\right)}=\frac{\Gamma(z+w+(N-1) \theta+1)}{\Gamma(z+n+(N-1) \theta+1) \Gamma(w+1-n)} \\
=\frac{\Gamma(z+w+(N-1) \theta+1)}{\Gamma(w+1) \Gamma(z+(N-1) \theta-q)} \cdot \frac{\Gamma(w+1) \Gamma(z+(N-1) \theta-q)}{\Gamma(w-n+1) \Gamma(z+(N-1) \theta+1+n)} . \quad(3.32)
\end{gathered}
$$

Это преобразование корректно, поскольку $\Gamma(z+(N-1) \theta-q)$ определено при достаточно больших $\operatorname{Re} z$. Первая дробь в (3.32) не зависит от $n$ и имеет, самое большее, полиномиальный рост по z. Что же касается второй дроби в (3.32), ее можно записать как

$$
\begin{aligned}
& \frac{w(w-1) \cdots(w-n+1)}{(z+(N-1) \theta-q)(z+(N-1) \theta-q+1) \cdots(z+(N-1) \theta-q+n-1)} \\
& \times \frac{1}{(z+(N-1) \theta-q+n) \cdots(z+(N-1) \theta-q+n+1) \cdots(z+(N-1) \theta+n)} \\
& \quad=(-1)^{n} \prod_{i=0}^{n-1} \frac{-w+i}{z+(N-1) \theta-q+i} \cdot \prod_{j=0}^{q} \frac{1}{z+(N-1) \theta-q+n+j} \cdot
\end{aligned}
$$

Беря Rez достаточно большим, получаем

$$
\left|\frac{-w+i}{z+(N-1) \theta-q+i}\right| \leqslant 1, \quad j=0, \ldots, n-1 .
$$

Следовательно, абсолютная величина первого произведения в (3.33) не превосходит 1. Наконец, второе произведение в (3.33) убывает как $n^{-q-1}$ (равномерно по $z)$. Это доставляет необходимую оценку на шаге 2.

Редукция к (3.6). Предыдущее рассуждение позволяет нам предположить, что $z, w$ суть целые неотрицательные числа. С этого места мы считаем, что $z=r \in \mathbb{Z}_{+}, w=s \in \mathbb{Z}_{+}$.

ЛЕмма 3.3. Зафиксируем $z=r \in \mathbb{Z}_{+}, w=s \in \mathbb{Z}_{+} u \nu \in \operatorname{SGN}(N)$, u пусть $z^{\prime}, w^{\prime}$ пробегают $\mathbb{C}$. Тогда

(i) величина $M_{N}\left(\nu \mid z, z^{\prime}, w, w^{\prime} ; \theta\right)$, определенная в (2.1), может бьть записана как

$$
\begin{aligned}
M_{N}\left(\nu \mid z, z^{\prime}, w, w^{\prime} ; \theta\right)=\operatorname{Dim}_{N}(\nu ; \theta) \operatorname{Dim}_{N}^{\prime}(\nu ; \theta) & \\
& \times \prod_{i=1}^{N} \frac{\Gamma(z+w+(i-1) \theta+1) \Gamma((i-1) \theta+1)}{\Gamma\left(z-\nu_{i}+(i-1) \theta+1\right) \Gamma\left(w+\nu_{i}+(N-i) \theta+1\right)} \\
& \times \prod_{i=1}^{N} \frac{\Gamma\left(-z^{\prime}+\nu_{i}-(i-1) \theta\right)}{\Gamma\left(-z^{\prime}-w-(i-1) \theta\right)} \cdot \frac{\Gamma\left(-w^{\prime}-\nu_{i}-(N-i) \theta\right)}{\Gamma\left(-w^{\prime}-z-(N-i) \theta\right)} \\
& \times \prod_{i=1}^{N} \frac{\Gamma\left(-z^{\prime}-w^{\prime}-z-w-(i-1) \theta\right)}{\Gamma\left(-z^{\prime}-w^{\prime}-(i-1) \theta\right)} ;
\end{aligned}
$$

(ii) эта величина есть рациинальная функция от $\left(z^{\prime}, w^{\prime}\right)$;

(iii) она обращцается в нуль при нарушении неравенства $\nu_{1} \leqslant r$ или $\nu_{N} \geqslant-s$. 
ДокАЗАТЕЛьСтво. (i) Чтобы преобразовать (2.1) в (3.35), надо применить тождество

$$
\frac{\Gamma(m+x)}{\Gamma(n+x)}=(-1)^{m+n} \frac{\Gamma(1-n-x)}{\Gamma(1-m-x)}, \quad m, n \in \mathbb{Z} .
$$

(ii) Параметры $z^{\prime}, w^{\prime}$ входят только в последние два произведения в (3.35). Ясно, что отношения гамма-множителей в этих произведениях суть рациональные функции от $\left(z^{\prime}, w^{\prime}\right)$ : здесь мы пользуемся тем, что $\nu_{i}, z, w$ суть целые числа.

(iii) Это следует из рассмотрения гамма-множителей в знаменателе первого произведения по $i=1, \ldots, N$. В самом деле, для $i=1$ член

$$
\left(\Gamma\left(z-\nu_{i}+(i-1) \theta+1\right)\right)^{-1}=\left(\Gamma\left(r-\nu_{1}+1\right)\right)^{-1}
$$

обращается в нуль при $\nu_{1}>r$. Аналогично, для $i=N$ член

$$
\left(\Gamma\left(w+\nu_{i}+(N-i) \theta+1\right)\right)^{-1}=\left(\Gamma\left(s+\nu_{N}+1\right)\right)^{-1}
$$

обращается в нуль при $\nu_{N}<-s$.

В силу утверждения (iii) леммы 3.3 бесконечные суммы в (3.9) и (3.10) являются в действительности конечными, и в силу утверждения (ii) обе части формул (3.9) и (3.10) рациональны по $\left(z^{\prime}, w^{\prime}\right)$. Поэтому достаточно проверить тождества $(3.9)$ и (3.10) в случае, когда каждый из параметров $z^{\prime}, w^{\prime}$ принимает какое-нибудь счетное множество значений. Согласно (3.8), мы специализируем $z^{\prime}=-s-k \theta, w^{\prime}=-r-l \theta$, где предполагается, что $k, l-$ достаточно большие положительные целые числа. Заметим, что тогда выражение (3.35) корректно определено.

Следовательно, мы свели нашу задачу к проверке соотношения (3.6), поскольку для мер $\widetilde{M}_{N}(\cdot \mid \lambda)$ тождества $(3.9)$ и (3.10) очевидны.

Вычисление $\hat{q}(\nu, \lambda ; \theta)$ с помощью соотношения дуальности для косых функций Джека. Мы предполагаем, что $\lambda$ задано формулой (3.5). Теперь наша цель - вычислить $\widetilde{M}_{N}(\nu \mid \lambda)=\hat{q}(\nu, \lambda ; \theta)$ и затем проверить соотношение $(3.6)$.

Заметим сначала, что $\hat{q}(\nu, \lambda ; \theta)$ обращается в нуль при нарушении неравенства $\nu_{1} \leqslant r$ или $\nu_{N} \geqslant-s$.

Затем заметим, что обе части в (3.6) не изменятся, если прибавить фиксированное целое число $m \mathrm{~K} z, z^{\prime},-w,-w^{\prime}$ и ко всем $\nu_{i}$. В самом деле, для правой части, которая дается выражением (3.35), это очевидно. Далее, в силу (1.1) коэффициенты $q(\mu, \nu ; \theta)$ инвариантны относительно преобразования $\mu \mapsto \mu+m$, $\nu \mapsto \nu+m$, и, значит, коэффициенты $\hat{q}(\nu, \lambda ; \theta)$ также инвариантны.

Стало быть, не нарушая общности, мы можем положить $s=0$ и можем считать $\lambda$ и $\nu$ разбиениями (эквивалентно, диаграммами Юнга), такими, что $\nu \subseteq \lambda=\left(r^{k}\right)$. Более того, мы знаем, что $\ell(\nu) \leqslant N$, где $\ell(\nu)-$ длина разбиения $\nu$. Нам понадобится различать многочлены Джека с конечным числом переменных и симметрические функции Джека с бесконечным числом переменных. Первые индексируются сигнатурами, а вторые - диаграммами Юнга (более общо, косыми диаграммами Юнга). Условимся обозначать симметрические функции Джека символом $\mathscr{P}$.

В силу известной формулы

$$
\mathscr{P}_{\lambda}\left(x_{1}, x_{2}, \ldots ; y_{1}, y_{2}, \ldots\right)=\sum_{\nu \subseteq \lambda} \mathscr{P}_{\lambda / \nu}\left(y_{1}, y_{2}, \ldots\right) \mathscr{P}_{\nu}\left(x_{1}, x_{2}, \ldots\right)
$$


и определения коэффициентов $\hat{q}(\cdot, \cdot)$ мы получаем

$$
\hat{q}(\nu, \lambda ; \theta)=\frac{\mathscr{P}_{\nu}\left(1^{N}\right) \mathscr{P}_{\lambda / \nu}\left(1^{k+l-N}\right)}{\mathscr{P}_{\lambda}\left(1^{k+l}\right)},
$$

где мы используем обозначение $1^{N}=(\underbrace{1, \ldots, 1}_{N}, 0,0, \ldots)$.

Величины $\mathscr{P}_{\nu}\left(1^{N}\right)$ и $\mathscr{P}_{\lambda}\left(1^{k+l}\right)$ находятся по известной формуле, см. (3.55) ниже. Проблема связана с $P_{\lambda / \nu}\left(1^{k+l-N}\right)$, поскольку формула (3.55) не переносится на общие косые функции Джека. Однако, как мы сейчас увидим, $P_{\lambda / \nu}\left(1^{k+l-N}\right)$ удается вычислить благодаря тому, что $\lambda$ имеет очень специальный вид (3.5).

Нам нужны некоторые обозначения. Зафиксируем прямоугольную диаграмму Юнга $\left(r^{k}\right)$. Для диаграммы Юнга $\nu \subseteq\left(r^{k}\right)$ пусть $\nu^{*}-$ результат поворота на $180^{\circ}$ косой диаграммы $\left(r^{k}\right) / \nu$; это снова обычная диаграмма Юнга. Эквивалентное определение:

$$
\nu^{*}=\left(r-\nu_{k}, \ldots, r-\nu_{1}\right) .
$$

Далее, пусть $1 / b_{\nu}(\theta)$ обозначает квадрат нормы функции $\mathscr{P}_{\nu}$ относительно стандартного скалярного произведения, зависящего от $\theta$, в алгебре симметрических функций (имеется в виду то скалярное произведение, в котором функции Джека образуют ортогональный базис; в обозначениях $[16, \S \mathrm{VI} .10]$ это $\left.\langle\cdot, \cdot\rangle_{1 / \theta}\right)$.

Лемма 3.4. Пусть $\nu и \mu-$ две диаграммь Юнга, такие, ито $\nu \subseteq \mu \subseteq\left(r^{k}\right)$. Тогда справедливо соотношение дуальности

$$
\mathscr{P}_{\mu / \nu}=\operatorname{const} \mathscr{P}_{\nu^{*} / \mu^{*}}
$$

гวe

$$
\text { const }=\frac{b_{\nu}(\theta) b_{\nu^{*}}(\theta)}{b_{\mu}(\theta) b_{\mu^{*}}(\theta)} \frac{\left\|P_{\nu}\right\|_{k, \theta}^{2}}{\left\|P_{\mu}\right\|_{k, \theta}^{2}} .
$$

Здесь через $\|\cdot\|_{k, \theta}$ мы обозначаем норму в гильбертовом пространстве на mоре $\mathbb{T}^{k}$ по отношению $\kappa$ мере $(k !)^{-1} \prod_{i<j}\left|u_{i}-u_{j}\right|^{2 \theta} d u$, где du есть нормированная инвариантная мера.

КомментАРии. Этот факт был доказан Ноуми [19]. Он также содержится у Райнса [25], см. там $\$ 2$ и особенно следствие 2.8. И Ноуми, и Райнс в действительности работали в более общем контексте симметрических функций Макдональда. Отметим, что в простейшем случае симметрических функций Шура $(\theta=1)$ константа равна 1 , и дуальность $(3.42)$ становится почти очевидной, если использовать комбинаторное представление косых функций Шура через таблицы Юнга. Наконец, заметим, что $\left\|P_{\nu}\right\|_{k, \theta}=\left\|P_{\nu^{*}}\right\|_{k, \theta}$, откуда следует, что константа переходит в обратную величину при преобразовании $(\mu, \nu) \mapsto\left(\nu^{*}, \mu^{*}\right)$, как это и должно быть.

ДокАЗАТЕЛьство. Мы воспроизведем, слегка изменив обозначения, рассуждение Райнса, идея которого весьма проста. Если задана симметрическая функция $F$, то пусть $\left[F: \mathscr{P}_{\kappa}\right]$ обозначает соответствующий коэффициент в ее разложении по базису $\left\{\mathscr{P}_{\kappa}\right\}$.

Дуальность (3.42) эквивалентна равенству

$$
\left[\mathscr{P}_{\mu / \nu}: \mathscr{P}_{\kappa}\right]=\operatorname{const}\left[\mathscr{P}_{\nu^{*} / \mu^{*}}: \mathscr{P}_{\kappa}\right],
$$


где $\kappa-$ произвольная диаграмма Юнга. По хорошо известному свойству симметрических функций Джека (3.44) эквивалентно равенству

$$
\frac{b_{\nu}(\theta) b_{\kappa}(\theta)}{b_{\mu}(\theta)}\left[\mathscr{P}_{\nu} \mathscr{P}_{\kappa}: \mathscr{P}_{\mu}\right]=\mathrm{const} \frac{b_{\mu *}(\theta) b_{\kappa}(\theta)}{b_{\nu}^{*}(\theta)}\left[\mathscr{P}_{\mu^{*}} \mathscr{P}_{\kappa}: \mathscr{P}_{\nu^{*}}\right] \text {. }
$$

Заметим, что все диаграммы $\mu, \nu, \mu^{*}, \nu^{*}$ имеют не более $k$ строк. Отсюда следует, что обе части в (3.44) обращаются в нуль при нарушении неравенства $\ell(\kappa) \leqslant k$. Следовательно, мы можем предполагать, что $\ell(\kappa) \leqslant k$. Но тогда мы можем вычислить обе части в $(3.45)$ в терминах скалярного произведения $(\cdot, \cdot)_{k, \theta}$ на торе $\mathbb{T}^{k}$. Мы получим, что (3.45) эквивалентно равенству

$$
\begin{aligned}
& \frac{b_{\nu}(\theta)}{b_{\mu}(\theta)\left\|P_{\mu}\right\|_{k, \theta}^{2}} \int_{\mathbb{T}^{k}} P_{\nu}(u) P_{\kappa}(u) \overline{P_{\mu}(u)} W_{k}(u ; \theta) d u \\
& \quad=\operatorname{const} \frac{b_{\mu *}(\theta)}{b_{\nu^{*}}(\theta)\left\|P_{\nu^{*}}\right\|_{k, \theta}^{2}} \int_{\mathbb{T}^{k}} P_{\mu^{*}}(u) P_{\kappa}(u) \overline{P_{\nu^{*}}(u)} W_{k}(u, \theta) d u,
\end{aligned}
$$

где использовано сокращенное обозначение $u=\left(u_{1}, \ldots, u_{k}\right)$.

Далее, для $u \in \mathbb{T}^{k}$

$$
\overline{P_{\mu}(u)}=\left(u_{1} \ldots u_{k}\right)^{-r} P_{\mu^{*}}(u), \quad \overline{P_{\nu^{*}}(u)}=\left(u_{1} \ldots u_{k}\right)^{-r} P_{\nu}(u)
$$

значит, интегралы в (3.46) идентичны. Это дает соотношение (3.46) и одновременно выражение для константы.

СледствиЕ 3.5. Пусть, как выше, $\lambda=\left(r^{k}\right) u \nu \subseteq \lambda$. Тогда

$$
\mathscr{P}_{\lambda / \nu}\left(1^{k+l-N}\right)=\frac{b_{\nu}(\theta) b_{\nu^{*}}(\theta)}{b_{\lambda}(\theta)} \frac{\left\|P_{\nu}\right\|_{k, \theta}^{2}}{\left\|P_{\lambda}\right\|_{k, \theta}^{2}} \mathscr{P}_{\nu^{*}}\left(1^{k+l-N}\right) .
$$

Комбинируя это с (3.40), получаем далее

СЛЕДСТВИЕ 3.6. Пусть $\lambda=\left(r^{k}\right) u \nu \subseteq \lambda, \ell(\nu) \leqslant N$. Тогда

$$
\hat{q}(\nu, \lambda ; \theta)=\frac{b_{\nu}(\theta) b_{\nu^{*}}(\theta)}{b_{\lambda}(\theta)} \frac{\left\|P_{\nu}\right\|_{k, \theta}^{2}}{\left\|P_{\lambda}\right\|_{k, \theta}^{2}} \frac{\mathscr{P}_{\nu}\left(1^{N}\right) \mathscr{P}_{\nu^{*}}\left(1^{k+l-N}\right)}{\mathscr{P}_{\lambda}\left(1^{k+l}\right)} .
$$

Окончание доказательства. Напомним, что нам остается доказать, что

$$
\hat{q}(\nu, \lambda ; \theta)=\left.M_{N}\left(\nu \mid z, z^{\prime}, w, w^{\prime} ; \theta\right)\right|_{z=r, z^{\prime}=-k \theta, w=0, w^{\prime}=-r-l \theta} .
$$

Здесь $\lambda=\left(r^{k}\right), \nu \subset\left(r^{k}\right)$, и мы предполагаем, что $\ell(\nu) \leqslant N$ и, более того, $k, l$ настолько велики, что $N \leqslant \min (k, l)$.

Для левой части в (3.50) мы используем формулу (3.49) следствия 3.6. Все величины, входящие в (3.49), могут быть явно вычислены с помощью следующих формул.

- Обобщенный символ Похгаммера. Если $\nu$ - диаграмма Юнга, $X$ - переменная и $N \geqslant \ell(\nu)$, положим

$$
(X)_{\nu, \theta}=\prod_{(i j) \in \nu}(X+(j-1)-(i-1) \theta)=\prod_{i=1}^{N} \frac{\Gamma\left(X-(i-1) \theta+\nu_{i}\right)}{\Gamma(X-(i-1) \theta)}
$$


- Крюковые произведения. Если $\nu$ - диаграмма Юнга, положим

$$
H(\nu ; \theta)=\prod_{\square \in \nu}(a(\square) \theta+l(\square) \theta+1), \quad H^{\prime}(\nu ; \theta)=\prod_{\square \in \nu}(a(\square)+l(\square) \theta+\theta),
$$

где произведения берутся по клеткам $\square$ диаграммы $\nu$ и символы $a(\square)$ и $l(\square)$ обозначают «длину руки» и «длину ноги» соответственно ([16, Ch. VI, (6.14)]). Имеем (см. [16, Ch. VI, (10.20)])

$$
\begin{array}{rlrl}
H(\nu ; \theta) & =\frac{(N \theta+1-\theta)_{\nu, \theta}}{\operatorname{Dim}_{N}(\nu ; \theta)}, & & N \geqslant \ell(\nu), \\
H^{\prime}(\nu ; \theta) & =\frac{(N \theta)_{\nu, \theta}}{\operatorname{Dim}_{N}^{\prime}(\nu ; \theta)}, & N \geqslant \ell(\nu) .
\end{array}
$$

- Формула для $b_{\nu}(\theta)$. Если $\nu$ - диаграмма Юнга, то (см. [16, Ch. VI, (10.16)])

$$
b_{\nu}(\theta)=\frac{H^{\prime}(\nu ; \theta)}{H(\nu ; \theta)} .
$$

- Значение функции $\mathscr{P}_{\nu}$ в $\left(1^{N}\right)$. Если $\nu$ - диаграмма Юнга с $\ell(\nu) \leqslant N$, то

$$
\mathscr{P}_{\nu}\left(1^{N} ; \theta\right)=\operatorname{Dim}_{N}^{\prime}(\nu ; \theta)
$$

(эта формула может быть получена вырождением из [16, Example VI.6.4]).

- Формула для $\left\|P_{\nu}\right\|_{k, \theta}^{2}$. Если $\nu$ - диаграмма Юнга с $\ell(\nu) \leqslant k$ (или сигнатура из $\mathrm{SGN}(k))$, то (см. [16, ch. VI, (10.38)])

$$
\frac{\left\|P_{\nu}\right\|_{k, \theta}^{2}}{\left\|P_{\varnothing}\right\|_{k, \theta}^{2}}=\frac{\operatorname{Dim}_{k}^{\prime}(\nu ; \theta)}{\operatorname{Dim}_{k}(\nu ; \theta)} .
$$

Теперь мы располагаем всем необходимым для доказательства формулы (3.50). Предположим сначала, что $\nu-$ пустая диаграмма, $\nu=\varnothing$. Тогда в формулах возникает ряд сокращений, и мы проверяем, что обе части в (3.50) равны

$$
\frac{(l \theta)_{\pi}}{((k+l) \theta)_{\pi}}, \quad \pi:=\left(r^{N}\right) .
$$

Остается показать, что

$$
\frac{\hat{q}(\nu, \lambda ; \theta)}{\hat{q}(\varnothing, \lambda ; \theta)}=\frac{\left.M_{N}\left(\nu \mid z, z^{\prime}, w, w^{\prime} ; \theta\right)\right|_{z=r, z^{\prime}=-k \theta, w=0, w^{\prime}=-r-l \theta}}{\left.M_{N}\left(\varnothing \mid z, z^{\prime}, w, w^{\prime} ; \theta\right)\right|_{z=r, z^{\prime}=-k \theta, w=0, w^{\prime}=-r-l \theta}} .
$$

Используя формулы (3.51)-(3.56), мы проверяем, что левая часть в (3.50) равна

$$
\operatorname{Dim}_{N}(\nu ; \theta) \operatorname{Dim}_{N}^{\prime}(\nu ; \theta) \frac{(L-N)_{\nu^{*}}(k \theta)_{\nu}}{(k+1-\theta)_{\nu^{*}}((N-1) \theta+1)_{\nu}} .
$$

Используя тот факт, что

$$
\nu^{*}=(\underbrace{r, \ldots, r}_{k-N}, \widetilde{\nu}_{1}, \ldots, \widetilde{\nu}_{N}), \quad \widetilde{\nu}:=\left(r-\nu_{N}, \ldots, r-\nu_{1}\right),
$$

мы далее преобразуем (3.59) в

$$
\operatorname{Dim}_{N}(\nu ; \theta) \operatorname{Dim}_{N}^{\prime}(\nu ; \theta) \frac{(k \theta)_{\nu}(l \theta)_{\widetilde{\nu}}((N-1) \theta+1)_{\pi}}{((N-1) \theta+1)_{\nu}((N-1) \theta+1)_{\widetilde{\nu}}(l \theta)_{\pi}} .
$$


Наконец, непосредственно проверяется, что правая часть в (3.50) также приводит к (3.61). На этом завершается доказательство теоремы 2.3.

\section{§4. Связь с формулой суммирования Густафсона}

Здесь мы намереваемся показать, что теорема 2.3 есть частный случай гипергеометрической формулы суммирования, принадлежащей Густафсону1). Мы объясняем также, как вывести из формулы Густафсона соотношение (1.7). Это показывает, что, в принципе, вся теория может быть основана на формуле Густафсона, без обращения к многочленам Джека. Однако приведенное ниже рассуждение выглядит как манипуляция формулами, и необходима дальнейшая работа, чтобы раскрыть истинный механизм этого подхода.

Мы сформулируем результат Густафсона (см. [10, Theorem 1.11]) в слегка измененных обозначениях.

ТеОремА ГУСТАФСОНА. Пусть $K=1,2, \ldots u \varkappa=\left(\varkappa_{j}\right)$ nробегаеm $\mathbb{Z}^{K}$. Пусть $\alpha=\left(\alpha_{r}\right) \in \mathbb{C}^{K+1}, \beta=\left(\beta_{r}\right) \in \mathbb{C}^{K+1} u \varepsilon=\left(\varepsilon_{j}\right) \in \mathbb{C}^{K}$ cymb napamempol. Предnoложим, что

(i) $\operatorname{Re}\left(\sum \beta_{r}-\sum \alpha_{r}\right)>K$,

(ii) числа $\varepsilon_{j}$, где $j=1, \ldots, K$, попарно различны,

(iii) числа $\alpha_{r}+\varepsilon_{j}$ и $1-\beta_{r}-\varepsilon_{j}$ не являются положительными цуельми ни для каких $r, j$.

Tогда

$$
\begin{aligned}
\sum_{\varkappa \in \mathbb{Z}^{K}} & \prod_{1 \leqslant i<j \leqslant K}\left(\frac{\left(\varkappa_{i}+\varepsilon_{i}\right)-\left(\varkappa_{j}+\varepsilon_{j}\right)}{\varepsilon_{i}-\varepsilon_{j}}\right) \prod_{r=1}^{K+1} \prod_{j=1}^{K} \frac{\left(\alpha_{r}+\varepsilon_{j}\right)_{\varkappa_{j}}}{\left(\beta_{r}+\varepsilon_{j}\right)_{\varkappa_{j}}} \\
= & \frac{\Gamma\left(-K+\sum_{r=1}^{K+1}\left(\beta_{r}-\alpha_{r}\right)\right) \prod_{r=1}^{K+1} \prod_{j=1}^{K} \Gamma\left(1-\alpha_{r}-\varepsilon_{j}\right) \Gamma\left(\beta_{r}+\varepsilon_{j}\right)}{\prod_{r, s=1}^{K+1} \Gamma\left(\beta_{r}-\alpha_{s}\right) \prod_{1 \leqslant i<j \leqslant K} \Gamma\left(1-\varepsilon_{i}+\varepsilon_{j}\right) \Gamma\left(1+\varepsilon_{i}-\varepsilon_{j}\right)},
\end{aligned}
$$

где для $a \in \mathbb{C} u n \in \mathbb{Z}$ положено

$$
(a)_{n}=\frac{\Gamma(a+n)}{\Gamma(a)}= \begin{cases}a(a+1) \ldots(a+n-1), & n \geqslant 0, \\ \frac{1}{(a-1) \ldots(a-|n|)}, & n \leqslant 0 .\end{cases}
$$

Введем обозначение

$$
k_{j}=\varkappa_{j}+\varepsilon_{j}, \quad j=1, \ldots, K,
$$

и отметим, что

$$
(a)_{n}=(-1)^{n} \frac{\Gamma(1-a)}{\Gamma(1-a-n)} .
$$

Используя это, перепишем (4.1) как

$$
\sum_{\varkappa \in \mathbb{Z}^{K}} G^{\theta}(\varkappa ; \alpha, \beta, \varepsilon)=1,
$$

\footnotetext{
1)Густафсон отмечает, что его результат есть многомерный аналог ${ }_{2} H_{2}$-формулы Дуголла (0.1).
} 
где

$$
\begin{aligned}
& G^{\theta}(\varkappa ; \alpha, \beta, \varepsilon)=\prod_{1 \leqslant i<j \leqslant K}\left(k_{i}-k_{j}\right) \prod_{r=1}^{K+1} \prod_{j=1}^{K} \frac{1}{\Gamma\left(1-\alpha_{r}-k_{j}\right) \Gamma\left(\beta_{r}+k_{j}\right)} \\
& \times(-1)^{(K+1)\left(\varkappa_{1}+\cdots+\varkappa_{K}\right)} \frac{\prod_{r, s=1}^{K+1} \Gamma\left(\beta_{r}-\alpha_{s}\right) \prod_{1 \leqslant i<j \leqslant K} \Gamma\left(1-\varepsilon_{i}+\varepsilon_{j}\right) \Gamma\left(\varepsilon_{i}-\varepsilon_{j}\right)}{\Gamma\left(-K+\sum_{r=1}^{K+1}\left(\beta_{r}-\alpha_{r}\right)\right)} .
\end{aligned}
$$

ЛЕмма 4.1. Пусть $\nu \in \operatorname{SGN}(N), \mu \in \operatorname{SGN}(N-1) u \mu \prec \nu$. Введем сокращзеннье обозначения

$$
n_{i}=\nu_{i}-i \theta, \quad i=1, \ldots, N, \quad m_{i}=\mu_{i}-i \theta, \quad i=1, \ldots, N-1
$$

Tогда

$$
\begin{aligned}
q(\mu, \nu ; \theta) & =\prod_{1 \leqslant i<j \leqslant N} \frac{\Gamma\left(m_{i}-n_{j}\right)}{\Gamma\left(m_{i}-n_{j}+1-\theta\right)} \times \prod_{1 \leqslant i \leqslant j \leqslant N-1} \frac{\Gamma\left(n_{i}-m_{j}+\theta\right)}{\Gamma\left(n_{i}-m_{j}+1\right)} \\
& \times \prod_{1 \leqslant i<j \leqslant N} \frac{\Gamma\left(n_{i}-n_{j}+1-\theta\right)}{\Gamma\left(n_{i}-n_{j}+\theta\right)} \times \prod_{1 \leqslant i<j \leqslant N-1}\left(m_{i}-m_{j}\right) \times \frac{\Gamma(N \theta)}{(\Gamma(\theta))^{N}} .
\end{aligned}
$$

ДокАЗАТЕЛЬСтво. Это следует из формулы (1.6), формулы (3.55) (которая справедлива также для сигнатур) и известного явного выражения для коэффициентов в (1.6)

$$
\psi_{\nu / \mu}(\theta)=\prod_{1 \leqslant i \leqslant j<N} \frac{\left(\mu_{i}-\mu_{j}+(j-i) \theta+\theta\right)_{\mu_{j}-\nu_{j+1}}\left(\nu_{i}-\mu_{j}+(j-i) \theta+1\right)_{\mu_{j}-\nu_{j+1}}}{\left(\mu_{i}-\mu_{j}+(j-i) \theta+1\right)_{\mu_{j}-\nu_{j+1}}\left(\nu_{i}-\mu_{j}+(j-i) \theta+\theta\right)_{\mu_{j}-\nu_{j+1}}}
$$

Teopema 4.2. Пусть $\nu \in \operatorname{SGN}(N), \mu \in \operatorname{SGN}(N-1), \mu \prec \nu, u$ nycmb uucла $q(\mu, \nu ; \theta)$ заданы формулой (4.8). Тогда соотношение (1.7) может быть получено как частный случай тождества Густафсона (4.5)-(4.6).

ДоКАЗАТЕЛЬСТВО. Подставим в (4.6)

$$
K=N-1, \quad \varkappa=\mu, \quad \varepsilon_{j}=-j \theta, \quad \alpha_{r}=-n_{r}, \quad \beta_{r}=-n_{r}+1-\theta,
$$

где мы временно предполагаем, что $\mu-$ произвольный элемент решетки $\mathbb{Z}^{N-1}$. Условие (i) теоремы Густафсона принимает вид $1-N \theta>0$; поэтому мы предположим, что $\theta<1 / N$. От этого допущения затем легко избавиться ввиду аналитичности по $\theta$. Условие (ii) очевидно. Условие (iii) сводится к возможному исключению некоторого конечного числа значений параметра $\theta$, что также не представляет проблем. 
При специализации (4.10) выражение (4.6) принимает вид

$$
\begin{aligned}
& \prod_{1 \leqslant i<j \leqslant N-1}\left(m_{i}-m_{j}\right) \prod_{r=1}^{N} \prod_{j=1}^{N-1} \frac{1}{\Gamma\left(n_{r}-m_{j}+1\right) \Gamma\left(-n_{r}+m_{j}+1-\theta\right)} \\
& \times \prod_{r, s=1}^{N} \Gamma\left(n_{r}-n_{s}+1-\theta\right) \prod_{1 \leqslant i<j \leqslant N-1} \Gamma(1+(i-j) \theta) \Gamma((j-i) \theta) \\
& \times \frac{(-1)^{N\left(\mu_{1}+\cdots+\mu_{N-1}\right)}}{\Gamma(1-N \theta)} .
\end{aligned}
$$

Заметим теперь, что (4.11) обращается в нуль, если не выполнено условие $\mu \prec \nu$. В самом деле, множитель $1 / \Gamma\left(n_{r}-m_{j}+1\right)$ при $r=j$ сводится к $1 / \Gamma\left(\nu_{j}-\right.$ $\left.\mu_{j}+1\right)$, что обращается в нуль при нарушении неравенства $\nu_{j} \geqslant \mu_{j}$. Аналогично, множитель $1 / \Gamma\left(-n_{r}+m_{j}+1-\theta\right)$ при $r=j+1$ сводится к $1 / \Gamma\left(\mu_{j}-\nu_{j+1}+1\right)$, что обращается в нуль при нарушении неравенства $\mu_{j} \geqslant \nu_{j+1}$.

Таким образом, суммирование в (4.5) будет фактически происходить по сигнатурам $\mu \prec \nu$, как в (1.7). Наконец, непосредственно проверяется, что (4.11) совпадает с (4.8). Это завершает доказательство.

ТЕОрема 4.3. Утверждение теоремь 2.3 может быть получено как частный случай тождества Густафсона (4.5)-(4.6).

ДокАЗАТЕЛЬСтво. Рассуждение аналогично предыдущему. Мы должны проверить соотношение когерентности (0.9). Мы записываем (0.9) наподобие (1.7):

$$
\sum_{\nu \in \operatorname{SGN}(N): \mu \prec \nu} p\left(\mu, \nu \mid z, z^{\prime}, w, w^{\prime} ; \theta\right)=1,
$$

где

$$
p\left(\mu, \nu \mid z, z^{\prime}, w, w^{\prime} ; \theta\right)=\frac{q(\mu, \nu ; \theta) M_{N}\left(\nu \mid z, z^{\prime}, w, w^{\prime} ; \theta\right)}{M_{N-1}\left(\mu \mid z, z^{\prime}, w, w^{\prime} ; \theta\right)} .
$$

Отметим, что выражения (4.13) суть переходные вероятности в смысле Вершика и Керова, см. [26, 15].

Теперь выбираем подходящую специализацию параметров Густафсона:

$$
\begin{gathered}
K=N, \quad \varkappa=\nu \in \mathbb{Z}^{N}, \quad \varepsilon_{j}=-j \theta \quad(j=1, \ldots, N), \\
\alpha_{r}=-m_{r}+\theta, \quad \beta_{r}=-m_{r}+1 \quad(r=1, \ldots, N-1), \\
\alpha_{N}=-z+\theta, \quad \alpha_{N+1}=-z^{\prime}+\theta, \\
\beta_{N}=w+N \theta+1, \quad \beta_{N+1}=w^{\prime}+N \theta+1,
\end{gathered}
$$

и выписываем в этой специализации (4.6). Снова оказывается, что полученное выражение обращается в 0 , если $\nu$ не является сигнатурой, такой, что $\mu \prec \nu$, а если последнее условие выполнено, то наше выражение совпадает с (4.13).

\section{§5. Связь с интегралами типа Сельберга}

Как было показано в §3, применение теоремы Карлсона позволяет свести утверждение теоремы 2.3 к ее частному случаю, когда $z=r=1,2, \ldots$ и $w=0$. 
Теорема 5.1. Утверждение теоремь 2.3 в случае, когда $z=r=1,2, \ldots u$ $w=0$, эквивалентно следующей формуле:

$$
\begin{aligned}
& \frac{M_{N}\left(\nu \mid r, z^{\prime}, 0, w^{\prime} ; \theta\right)}{P_{\nu}(\underbrace{1, \ldots, 1}_{N} ; \theta)} \\
& \quad=\int_{1 \geqslant \beta_{1} \geqslant \cdots \geqslant \beta_{r} \geqslant 0} P_{\nu^{\prime}}\left(\frac{\beta_{1}}{1-\beta_{1}}, \ldots, \frac{\beta_{r}}{1-\beta_{r}} ; \theta^{-1}\right) \prod_{i=1}^{r}\left(1-\beta_{i}\right)^{N} M_{\infty}(d \beta) .
\end{aligned}
$$

Здесь $\nu$ - диаграмма Юнга, содержащаяся в $\left(r^{N}\right), \nu^{\prime}-$ транспонированная диаграмма и $M_{\infty}(d \beta)$ - нормированная мера на множестве $\Delta_{r}=\{\beta \mid 1 \geqslant$ $\left.\beta_{1} \geqslant \cdots \geqslant \beta_{r} \geqslant 0\right\}$ с плотностью

$$
\frac{1}{\operatorname{const}\left(r, \theta^{-1}, A, B\right)} \prod_{i=1}^{r} \beta_{i}^{A-1}\left(1-\beta_{i}\right)^{B-1} \prod_{1 \leqslant i<j \leqslant R}\left(\beta_{i}-\beta_{j}\right)^{2 / \theta},
$$

где

$$
A=\left(z^{\prime}-r+1\right) \theta^{-1}, \quad B=\left(w^{\prime}+1\right) \theta^{-1} .
$$

ИДЕЯ ДОКАЗАТЕЛЬСТВА. В случае $\theta=1$ этот результат был впервые получен Керовым [12]. Его доказательство переносится на случай общего $\theta$, но вычисления становятся более трудоемкими. Основным моментом является наличие биективного соответствия $\left\{M_{N}\right\} \leftrightarrow M_{\infty}$ между когерентными системами $\left\{M_{N}\right\}$, такими, что $M_{N}$ сосредоточено на неотрицательных сигнатурах $\nu$ с $\nu_{1} \leqslant r$, и вероятностными мерами $M_{\infty}$ на $\Delta_{r}$. При этом соответствии $M_{\infty}$ возникает как предел при больших $N$ образов мер $M_{N}$ относительно отображений

$$
\nu \mapsto\left(\nu_{1}^{\prime} / N, \ldots, \nu_{r}^{\prime} / N\right) \in \Delta_{r} .
$$

Обратно, для данной меры $M_{\infty}$ соответствующая когерентная система может быть выражена через $M_{\infty}$ посредством интегрального представления «типа Пуассона». В несколько ином контексте этот формализм описан в работах Керова [13] и [15]; см. также статью автора [22]. Мы используем также результат работы $[21]$.

Интеграл (5.1) очень похож на хорошо известный интеграл типа Сельберга с многочленом Джека (см. [11] или [16, Examples VI.9.3, VI.10.7]). Более того, интеграл (5.1) в действительности эквивалентен обычному интегралу типа Сельберга. Я не знаю, был ли известен этот факт Керову. Доказательство использует один прием, который можно извлечь из $[11, \S 8]$.

Как следствие мы получаем еще одно доказательство теоремы 2.3.

\section{ЛитеРАТУРА}

1. Andrews G. E., Askey R., Roy R. Special Functions. Cambridge University Press, 1999.

2. Borodin A., Olshanski $G$. Harmonic functions on multiplicative graphs and interpolation polynomials. Electronic J. Comb., 7, paper \#R28 (2000); arXiv: math/9912124.

3. Borodin A., Olshanski G. Infinite random matrices and ergodic measures. Comm. Math. Phys., 223, 87-123 (2001); arXiv: math-ph/0010015.

4. Borodin A., Olshanski G. Harmonic analysis on the infinite-dimensional unitary group and determinantal point processes. Ann. Math., to appear; arXiv: math.RT/0109194. 
5. Borodin A., Olshanski G. Z-measures on partitions and their scaling limits. Europ. J. Combin, to appear; arXiv: math-ph/0210148.

6. Dougall J. On Vandermonde's theorem and some more general expansions. Proc. Edinburgh Math. Soc., 25, 114-132 (1907).

7. Dyson F. Statistical theory of the energy levels of complex systems I-III. J. Math. Phys., 3, 140-156, 157-165, 166-175 (1962).

8. Бейтмен $Г$., Эрдейи А. Высшие трансцендентные функции, т. I. Наука (Физматлит), M., 1973.

9. Евграфов М. А. Асимптотические оценки и целые функции. Физматгиз, М., 1962.

10. Gustafson R. A. Multilateral summation theorems for ordinary and basic hypergeometric series in $U(n)$. SIAM J. Math. Anal., 18, No. 6, 1576-1596 (1987).

11. Kadell K. W. J. The Selberg-Jack symmetric functions. Adv. Math., 130, 33-102 (1997).

12. Керов C. В. Многомерное гипергеометрическое распределение и характеры унитарной группы (рабочие заметки 1993). В сб.: Теория представлений, динамические системы, комбинаторные и алгоритмические методы, вып. 9 (под ред. А. М. Вершика), готовится к печати.

13. Kerov $S$. $V$. The boundary of Young lattice and random Young tableaux. In: Formal power series and algebraic combinatorics. DIMACS Ser. Discrete Math. Theoret. Comput. Sci., Vol. 24, Amer. Math. Soc., Providence, RI, 1996, pp. 133-158.

14. Kerov $S$. V. Анизотропные диаграммы Юнга и симметрические функции Джека. Функц. анализ и его прил., 34, вып. 1, 51-64 (2000).

15. Kerov $S$. $V$. Asymptotic representation theory of the symmetric group and its applications in analysis. Amer. Math. Soc., Providence, RI, 2003.

16. Macdonald I. G. Symmetric functions and Hall polynomials, 2nd edition. Oxford University Press, 1995.

17. Neretin $Y u$. A. Hua-type integrals over unitary groups and over projective limits of unitary groups. Duke Math. J., 114, 239-266 (2002).

18. Неретин Ю. А. Треугольники Релея и нематричная интерполяция матричных бетаинтегралов. Матем. сб., в печати; arXiv: math.CA/0301070.

19. Noumi $M$. Complementary duality for skew Macdonald polynomials. Preprint, November 2001.

20. Okounkov A., Olshanski G. Shifted Jack polynomials, binomial formula, and applications. Math. Res. Lett., 4, 69-78 (1997).

21. Okounkov A., Olshanski G. Asymptotics of Jack polynomials as the number of variables goes to infinity. Intern. Math. Res. Notices, No. 13, 641-682 (1998).

22. Olshanski $G$. The problem of harmonic analysis on the infinite-dimensional unitary group. J. Funct. Anal., to appear; arXiv: math.RT/0109193.

23. Olshanski G. An introduction to harmonic analysis on the infinite symmetric group. In: Asymptotic Combinatorics with Applications to Mathematical Physics (A. Vershik, ed.). Lect. Notes in Math., Vol. 1815, Springer-Verlag, 2003, pp. 127-160.

24. Pickrell D. Measures on infinite dimensional Grassmann manifold. J. Func. Anal., 70 , 323-356 (1987).

25. Rains E. M. $B C_{n}$ symmetric polynomials. Preprint, 2001; arXiv: math.QA/0112035.

26. Вершик A. М., Керов C. В. Локально полупростые алгебры. Комбинаторная теория и $K_{0}$-функтор. Итоги науки и техники. Современные проблемы математики. Новейшие достижения. т. 26, ВИНИТИ, М., 1985.

27. Vershik A. M., Kerov S. V. The Grothendieck group of the infinite symmetric group and symmetric functions with the elements of the $K_{0}$-functor theory of AF-algebras. In: Representation of Lie groups and related topics. Adv. Stud. Contemp. Math., Vol. 7 (A. M. Vershik and D. P. Zhelobenko, eds.), Gordon and Breach, (1990), pp. 36-114. 